\title{
Retrieval of macrophysical cloud parameters from MIPAS: algorithm description
}

\author{
J. Hurley, A. Dudhia, and R. G. Grainger \\ Atmospheric, Oceanic and Planetary Physics, Clarendon Laboratory, Department of Physics, Parks Road, Oxford, UK \\ Received: 16 August 2010 - Published in Atmos. Meas. Tech. Discuss.: 26 August 2010 \\ Revised: 29 March 2011 - Accepted: 3 April 2011 - Published: 7 April 2011
}

\begin{abstract}
The Michelson Interferometer for Passive Atmospheric Sounding (MIPAS) onboard ENVISAT has the potential to be particularly useful for studying high, thin clouds, which have been difficult to observe in the past. This paper details the development, implementation and testing of an optimal-estimation-type retrieval for three macrophysical cloud parameters (cloud top height, cloud top temperature and cloud extinction coefficient) from infrared spectra measured by MIPAS. A preliminary estimation of a parameterisation of the optical and geometrical filling of the measurement field-of-view by cloud is employed as the first step of the retrieval process to improve the choice of a priori for the macrophysical parameters themselves.

Preliminary application to single-scattering simulations indicates that the retrieval error stemming from uncertainties introduced by noise and by a priori variances in the retrieval process itself is small - although it should be noted that these retrieval errors do not include the significant errors stemming from the assumption of homogeneity and the non-scattering nature of the forward model. Such errors are preliminarily and qualitatively assessed here, and are likely to be the dominant error sources. The retrieval converges for $99 \%$ of input cases, although sometimes fails to converge for vetically-thin $(<1 \mathrm{~km})$ clouds. The retrieval algorithm is applied to MIPAS data; the results of which are qualitatively compared with CALIPSO cloud top heights and PARASOL cloud opacities. From comparison with CALIPSO cloud products, it must be noted that the cloud detection method used in this algorithm appears to potentially misdetect stratospheric aerosol layers as cloud.
\end{abstract}

This algorithm has been adopted by the European Space Agency's "MIPclouds" project.

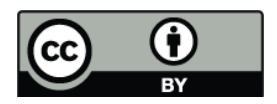

Correspondence to: J. Hurley

(hurley@atm.ox.ac.uk)

\section{Introduction}

Although much of atmospheric infrared remote sensing is based upon analysis of data to estimate constituent concentrations - where the presence of cloud particles in the measurements is treated as a source of error - it is possible to isolate measurements of cloud in order to determine the properties of clouds themselves. Clouds (especially high cloud such as cirrus) represent one of the largest uncertainties in climate studies (Intergovernmental Panel on Climate Change, 2008) - and in order to have reliable estimates of radiative forcing and climatic impact, accurate distributions of cloud frequencies and properties must be available. Satellite instruments provide an opportunity to study the properties of clouds on a global scale.

\subsection{Overview of MIPAS-ENVISAT}

The Michelson Interferometer for Passive Atmospheric Sounding (MIPAS) is an infrared limb-viewing instrument and was launched in March 2002 on the European Space Agency's Environmental Satellite (ENVISAT). The polar orbit of ENVISAT has large inclination, and enables global coverage pole-to-pole over a period of days, with an orbital repeat period of 35 days (European Space Agency, 2005).

MIPAS was designed to measure limb-emission spectra, primarily for retrieval of information upon trace gases such as $\mathrm{CO}_{2}$ (used to retrieve pressure and temperature), $\mathrm{O}_{3}, \mathrm{H}_{2} \mathrm{O}$, $\mathrm{HNO}_{3}, \mathrm{CH}_{4}, \mathrm{~N}_{2} \mathrm{O}$ and $\mathrm{NO}_{2}$, at a high spectral resolution in the near- to mid-infrared from $685 \mathrm{~cm}^{-1}$ to $2410 \mathrm{~cm}^{-1}$. In its initial phase, MIPAS operated at a spectral sampling of $0.025 \mathrm{~cm}^{-1}$, measuring spectra nominally every $3 \mathrm{~km}$ vertically in the troposphere down to approximately $6 \mathrm{~km}$. However, following persistent malfunctions in the smooth and consistent operation of the interferometer slide mechanism in early 2004, the sampling was decreased to $0.0625 \mathrm{~cm}^{-1}$ but the measurement frequency increased to nominally every

Published by Copernicus Publications on behalf of the European Geosciences Union. 
$1.5 \mathrm{~km}$ upward from approximately $4.5 \mathrm{~km}$ in the troposphere through the lower stratosphere (Mantovani, 2005). The MIPAS field-of-view is trapezoidal in the vertical, with a vertical extent varying between 3-4 km, depending upon definition. It has a characteristically wide horizontal field-of-view, extending approximately $200 \mathrm{~km}$ along the line-of-sight.

\subsection{Overview of clouds from satellites}

In this section, a very brief overview of the types of parameters which are typically used to describe clouds is given, along with a sampling of general characteristics of different instruments which demonstrate how certain types of observations are suitable for analysis of certain cloud properties (Sect. 1.2.1). Section 1.2.2 gives details of how instruments such as MIPAS tend to retrieve cloud properties.

\subsubsection{Generalities of cloud measurement}

Cloud properties fall loosely into two categories: macrophysical and microphysical. Macrophysical properties are the large-scale properties (i.e. bulk or extent), such as the altitude of a cloud, the physical depth and extent of a cloud, or are basic thermodynamic quantities, such as the temperature at the cloud top or the temperature structure within the cloud body. Microphysical parameters are, by opposition, those which relate to the small-scale of the cloud - such as the size and shape of cloud particles, and their distribution (which is often described in terms of water content), thus including properties such as number density, and influencing cloud optical depth, albedo, emissivity and transmissivity. Cloud extinction is strictly a combination of macrophysical and microphysical parameters as it is derived from both the physical extent of the cloud, as well as its absorption and scattering characteristics. However, for a model whereby there is no scattering, and a single homogeneous extinction characterising the bulk of the cloud mass, as is the case in this study, the extinction coefficient is designated as a macrophysical parameter.

Most of our knowledge on the microphysical properties of clouds come from in-situ measurements: predominantly by aircraft-mounted instruments. Satellite instruments are particularly well-suited to observing bulk cloud properties, such as average cloud top height and temperature, not least because of the large-scale geographical regions they survey.

As a general rule, limb-viewing instruments are competent at retrieving vertically-dependent parameters (such as cloud top height/pressure or cloud depth/extent) with great accuracy, although have poorer horizontal-resolving potential. They are able to detect even clouds having thin opacities of less than 0.01 due to the inherently long limb pathlength. On the contrary, traditional nadir-viewing instruments generally suffer from poor vertical resolution when retrieving atmospheric temperature and composition from which cloud top temperatures (and hence cloud top heights/pressures) are derived, and are limited to thicker clouds, but have very good horizontal resolution. It should be emphasized that nadir instruments have a distinct advantage over their limb-viewing counterparts in terms of compilation of climatologies, as their (typically) larger swath widths allow for greater coverage, implying better spatial resolution and statistical significance of averaged properties. Furthermore, certain current nadir instruments, such as CloudSat and CALIPSO (see Sect. 1.2.2) have vertical resolutions comparable to limb instruments.

Different spectral ranges are sensitive to different cloud properties. For instance, microwave instruments often are not sensitive to small ice cloud particles found in thin cirrus since such long wavelengths do not cause much scattering from typical ice particles, whereas visible and infrared instruments are often limited to the first layer of cloud encountered and unable to measure below as typical clouds will be opaque to radiation at these wavelengths (e.g. ESA's Living Planet website, 2010).

As different instruments are sensitive to only certain cloud properties, due to inherent differences in sensitivities in spectral ranges, viewing geometries and so forth, it is thus important to choose to retrieve cloud properties appropriate to the satellite instrument's capabilities.

There have been many studies on clouds (both missions and case studies) over the years producing cloud products: for example, by Barton (1983), Warren et al. (1985), Woodbury and McCormick (1983), Prabhakara et al. (1988), Wylie and Menzel (1989), Wylie et al. (1994), and King et al. (2010). Current instruments/projects/mission include the Stratospheric Aerosol and Gas Experiment SAGE (e.g. SAGE-III-ATBD-Team, 2002), CloudSat (e.g. Stephens et al., 2002), the Odin-submillimetre radiometer (SMR; e.g. Rydberg et al., 2009), the High Resolution Infrared Radiation Sounder HIRS instrument (e.g. Wylie et al., 2005), the Microwave Limb Sounder MLS (e.g. Wu et al., 2008), Polarization and Anisotropy of Reflectances for Atmospheric Sciences coupled with Observations from a Lidar PARASOL (e.g. Fougnie et al., 2007), the International Satellite Cloud Climatology Project ISCCP (e.g. ISCCP, 2006), Cloud-Aerosol Lidar and Infrared Pathfinder Satellite Observations CALIPSO (e.g. Winker et al., 2009), the GRAPE project (e.g. Thomas et al., 2010), and the GEWEX project (e.g. Stubenrauch et al., 2009).

However, past and current satellite cloud detection algorithms often miss much thin cloud - and hence conventional cloud climatologies and inventories are in no way complete with respect to high thin cloud such as cirrus (Wylie et al., 2005). In fact, limb-viewing has not been traditionally used for cloud measurements, although the new generation of instruments now are beginning to include cloud as a product on limb-viewing platforms. In the past, limb instruments tended to target atmospheric composition for which cloud detection is the only requirement. Given that MIPAS should be quite sensitive to high, thin cloud, if an appropriate detection 
method is employed it is a natural candidate to contribute climatological information about these clouds.

\subsubsection{Specifics of high cloud measurement by MIPAS- like instruments}

Retrieval of cloud parameters from instruments such as MIPAS, although highly instrument-specific, are often dependent upon cloud-detection algorithms as estimators of cloud location (cloud top height/pressure/depth), and as selectors of data upon which retrieval schemes are run. Sometimes retrieval algorithms operationally process all data without filtering or detecting, even though retrievals are computationally expensive, and cloud detection methods provide a useful sub-filtering for efficient processing. Generally, cloud detection methods for IR limb-viewing (such as MIPAS) or solar occultation instruments are based upon (1) a threshold upon, or discontinuity in, some property which varies in the presence of cloud, or (2) a contrast in spectral structure.

The Limb Infrared Monitor of the Stratosphere (LIMS), a limb sounder flown onboard the Nimbus-7 satellite from 1978 to 1979 , was designed to make measurements of thermal IR emission in six bands to determine vertical profiles of $\mathrm{CO}_{2}, \mathrm{O}_{3}, \mathrm{HNO}_{3}, \mathrm{H}_{2} \mathrm{O}$, and $\mathrm{NO}_{2}$. LIMS detected cloud by considering the vertical gradient of the retrieved ozone mixing ratio and the minimum mixing ratio of the retrieved ozone. When the apparent mixing ratio was less than $0.5 \mathrm{ppmv}$ and the mixing ratio fraction was greater than 0.25 , cloud was detected. However, this detection method failed to detect some cloud which then was identified manually or with the aid of variance exceedence criteria from the level 3 mapping algorithm (which uses all the retrieved profiles along an orbit to map out the horizontal distribution of the retrieved parameters). LIMS retrieved cloud top height as an operational product (e.g. NASA, 2007).

The Atmospheric Trace Molecule Spectroscopy (ATMOS) Experiment was a Fourier-transform IR spectrometer flown three times the Space Shuttle between 1985 and 1994, using solar occultation. The instrument recorded solar transmission spectra over a wide range of wavelengths between 600 $4750 \mathrm{~cm}^{-1}$ at $0.01 \mathrm{~cm}^{-1}$ resolution. The four bandpasses most sensitive to cloud were filters $1\left(600-1200 \mathrm{~cm}^{-1}\right)$, $4\left(3150-4800 \mathrm{~cm}^{-1}\right), 9\left(600-2000 \mathrm{~cm}^{-1}\right)$ and $12(600-$ $\left.1400 \mathrm{~cm}^{-1}\right)$ - and cloud detection was based upon the principle that a sharp reduction in average transmission across microwindows located at $831 \mathrm{~cm}^{-1}, 957 \mathrm{~cm}^{-1}$ and $1204 \mathrm{~cm}^{-1}$ (having minimal gas absorption) indicated cloud presence. Appropriate thresholds were chosen to identify different cloud types. ATMOS used a model of cloud spectral transmission in the Christiansen bands, parametrised by a range of ice, aerosol and atmospheric gas optical depths to retrieve cloud extinction for ice clouds (Kahn et al., 2002).

The Improved Stratospheric and Mesospheric Sounder (ISAMS) was an IR radiometer built at Oxford University, and it operated onboard the Upper Atmosphere Research
Satellite (UARS) from 1991 to 1992 . It observed thermal emission from the Earth's limb using pressure modulating radiometry to measure vertical profiles of temperature mixing ratios of $\mathrm{CO}, \mathrm{H}_{2} \mathrm{O}, \mathrm{CH}_{4}, \mathrm{O}_{3}, \mathrm{HNO}_{3}, \mathrm{~N}_{2} \mathrm{O}_{5}, \mathrm{NO}, \mathrm{NO}_{2}$, $\mathrm{N}_{2} \mathrm{O}$ and aerosol extinction. Detection was carried out by thresholding retrieved very large values of aerosol extinction, and retrieval of cloud extinction and top height were accomplished by inverting emission measurements to get extinction information, using transmission look-up-tables (Taylor et al., 1993).

The Halogen Occultation Experiment (HALOE) was an IR solar occultation instrument aboard the Upper Atmospheric Research Satellite. HALOE measured aerosol extinction at four wavelengths $(2.45,3.46,5.26$ and $10.0 \mu \mathrm{m})$ and detected cloud in several ways. Firstly, the behaviour of the extinction was represented as a normalised variance and cloud was detected whenever the extinction fell beyond a certain threshold. Secondly, sharp changes in the vertical extinction profile were noted by analysing the vertical extinction gradient, whereby large gradients indicated cloud presence. Finally, simple thresholding on the registered extinctions themselves was used to identify cloud presence with large extinctions (Hervig and Deshler, 2002; Hervig and et al., 1998).

The Cryogenic Limb Array Etalon Spectrometer (CLAES) experiment was an IR limb emission spectrometer onboard the Upper Atmospheric Research Satellite. It registered in the spectral range of $3.5-12.9 \mu \mathrm{m}$ in order to measure temperature profiles, and concentrations of $\mathrm{O}_{3}, \mathrm{CH}_{4}, \mathrm{H}_{2} \mathrm{O}, \mathrm{NO}_{\mathrm{x}}$, and other important species including CFCs and aerosols in the stratosphere. CLAES cloud detection used the $12.8 \mu \mathrm{m}$ channel to empirically threshold to separate clouds from aerosol (CLAES, 2007). CLAES obtained cloud extinction values by inverting emission measurements using the continuum radiance at about $790 \mathrm{~cm}^{-1}$ (Mergenthaler et al., 1993).

The Cryogenic Infrared Spectrometers and Telescopes for the Atmosphere (CRISTA) was a limb scanning experiment flown on two space shuttle missions on the ASTRO-SPAS platform in 1994 and 1997. It measured thermal emissions in the spectral range of 4-71 $\mu \mathrm{m}$ in order to observe selected trace gases with high spatial resolution. CRISTA detected cloud by thresholding a ratio of the mean radiances in two MWs which responded differently to cloud presence. Using this detection method, CRISTA provided a rough measure of cloud top height (Spang et al., 2004).

The Atmospheric Chemistry Experiment (ACE) onboard SCISAT-1 launched in August 2003 employs a high resolution $\left(0.02 \mathrm{~cm}^{-1}\right)$ IR Fourier transform spectrometer operating from $2.2-13.3 \mu \mathrm{m}$. Cloud detection is achieved by monitoring the extinction of solar radiation at $1.02 \mu \mathrm{m}$ and $0.525 \mu \mathrm{m}$ as measured by two filtered imagers. ACE reports cloud top height, as determined from the detection process (Bernath, 2002), and extinction from values of atmospheric transmission obtained from the ratio of the incident solar radiation at the top of the atmosphere to that measured (Gilbert et al., 2007). 
The High Resolution Dynamics Limb Sounder (HIRDLS) is a multi-channel limb scanning IR radiometer onboard the Earth Observing System (EOS) Chemistry mission satellite AURA (launched in July 2004). It was designed to measure temperature in the upper atmosphere and to determine concentrations of $\mathrm{O}_{3}, \mathrm{H}_{2} \mathrm{O}, \mathrm{CH}_{4}, \mathrm{~N}_{2} \mathrm{O}, \mathrm{NO}_{2}, \mathrm{ClONO}_{2}$, $\mathrm{N}_{2} \mathrm{O}_{5}, \mathrm{HNO}_{3}$ and chlorofluorocarbon compounds CFC-11 and CFC-12, as well as the location of polar stratospheric clouds. HIRDLS uses finite channels to measure radiance spectra between $6 \mu \mathrm{m}$ and $18 \mu \mathrm{m}$ - and certain of these channels are particularly sensitive to cloud presence. The optically thin spectral channels, particularly channels 6,13 and 19 , clearly show enhanced radiance when a thick cloud is present - the cloud signature appears as a sharp increase in limb radiance due to the increased limb opacity. HIRDLS operationally reports cloud top pressures and extinctions by considering the radiance profiles in HIRDLS channel 6. For a single day's worth of radiance profiles, the clear sky radiance profile is calculated by an iterative technique for several latitude bands. The clear sky radiance profile is then used to identify the altitude level at which cloud radiance perturbations are first noted. Cloud top altitudes are then obtained by converting the cloud top pressures into height coordinates - however, since the cloud top altitudes are on a $1 \mathrm{~km}$ altitude grid, the cloud top pressure suffers from discretisation similar to that of the altitude gridding (Lambert et al., 1999; Khosravi et al., 2009). HIRDLS retrieves cloud/aerosol extinction by retrieving the full vertical temperature profile from a measurement scan in which cloud has been detected, and by using this temperature profile to retrieve extinction along the path (Massie et al., 2007).

Most of the IR limb and occultation instruments use simple threshold tests on radiance (CLAES and HIRDLS), transmission (ATMOS), extinction (ISAMS, HALOE, and ACE), or upon the vertical gradient of extinction (HALOE) and apparent trace gas concentrations such as ozone (LIMS) - however only CRISTA uses a threshold on a ratio of radiances. The act of detection yields cursory information on cloud frequency of occurrence and a preliminary measure of cloud top height. In terms of other retrieved cloud parameters, it should be noted that of the instruments discussed ATMOS, ISAMS, HALOE, HIRDLS, CLAES and ACE operationally retrieve extinction. These methods typically are non-scattering models, assuming homogeneity of the cloud studied, and usually report cloud top height/temperature "retrievals" based upon detection of cloud. The method described in this paper improves upon these in the sense that whilst cloud detection is used as a first guess of cloud location, a proper estimations scheme based on a physical model (albeit with several assumptions shared with past algorithms, as discussed in Sect. 3), is implemented to derive parameters having betterthan-limb-sampling vertical resolution.

This discussion is not complete without mention of the CALIPSO (Cloud-Aerosol Lidar and Infrared Pathfinder Satellite Observation) and PARASOL/POLDER (Polariza- tion and Directionality of the Earth's Reflectances) instruments, part of the A-train constellation. Even though these current instruments are not limb-viewing, they have comparable vertical resolutions to MIPAS (and to the other limbviewers discussed), and in fact represent the best candidate instruments for comparison with MIPAS cloud products. CALIPSO has an active lidar with passive visible and infrared imagers, which measure thin clouds and aerosol at extremely high spatial resolution (Winker et al., 2009). PARASOL/POLDER is a wide-field imaging radiometer/polarimeter measuring with 16 distinct viewing geometries (Leroy et al., 1997; Deschamps et al., 1994), which reports optical depths, derived from averaged optical depths over all of the measured directions/geometries. CALIPSO and PARASOL/POLDER cloud products have been briefly used to carry out a preliminary and qualitative comparison with the application of the presented algorithm to MIPAS data in Sect. 4.1.

\subsection{Cloud information from MIPAS}

There have been several attempts to retrieve cloud parameters from MIPAS spectra, including

- The Monte Carlo Cloud Scattering Forward Model (McCloudsFM): a multi-scattering model developed by Ewen (2005) to accurately model IR limb emission measurements of cirrus clouds, parameterised by effective radius, number density, cloud top height and cloud depth. The computational time associated with the retrieval, however, was prohibitively large, and could not be justified given assumptions made in scattering properties and a priori biases, since there are large degeneracies in the sets of cloud parameters which can produce similar radiance effects.

- Cloud top heights from cloud detection method: the Earth Observation Science Group at the University of Leicester produces near-real-time cloud top heights from MIPAS spectra from May 2008 onwards (Moore, 2008). The cloud top heights are retrieved using the operational cloud detection method called the Colour Index (CI) method (Spang, 2004) such that the amount of cloud occurring in a given FOV is roughly anticorrelated with the value of CI. Leicester reports the tangent altitude at which cloud is first encountered in the MIPAS scan pattern as the cloud top height.

- The Karlsruhe Optimised and Precise Radiative transfer Algorithm (KOPRA): provides accurate simulations of single-scattering clouds in a horizontally symmetric atmosphere, specific to MIPAS. KOPRA can simulate different cloud types, such as cirrus, liquid water clouds, and various types of PSCs - and thus can be used to retrieve the modelled microphysical properties (IMK, 2008) given appropriate a priori atmospheric information (e.g. Mendrok et al., 2007). 
To this end a more comprehensive and operational cloud parameter retrieval algorithm specific to MIPAS has been developed - and has been adopted as the macrophysical cloud parameter retrieval of the "MIPclouds" project (Spang et al., 2008). In this work, a non-scattering forward model of the radiation emitted by a cloud in the MIPAS FOV is described, in terms of three macrophysical parameters: cloud top height, top temperature and extinction coefficient corresponding to the limb path, which is dominated by the extinction of the cloud itself. The inverse problem is addressed using an adaptation of standard retrieval theory: a sequential retrieval in which the first guess and a priori are chosen using an estimate of cloud amount. It should be emphasised that the main purpose of this retrieval is to improve on previous limbsounder cloud top height retrievals which are based purely on detection by having a physically-based forward model, and attempting to retrieve cloud top height and temperature to better vertical resolution than the limb sampling itself.

\section{Algorithm description}

The retrieval of macrophysical parameters from a set of MIPAS spectra constituting a single limb-scan is a three-stage process applied independently in different spectral intervals ("microwindows"). These stages are:

1. isolating the continuum radiance from each spectrum;

2. retrieving the Cloud Effective Fraction (CEF) to locate the spectrum containing the cloud-top; and

3. retrieving the macrophysical parameters from this and vertically adjacent spectra within the limb scan pattern.

The results from each microwindow are combined to produce a best estimate of the parameters, and an associated error covariance.

\subsection{Microwindows}

Microwindows (MWs) are small subsets of the MIPAS spectrum of a few wavenumbers in width. A set of ten MWs have been selected from the MIPAS spectral range - and span the spectral region of $930-960 \mathrm{~cm}^{-1}$ (Table 1). They are selected using a modification of the MIPAS MW selection algorithm (Dudhia et al., 2002) optimised for a joint retrieval of continuum radiance and temperature, in which the MWs are ranked in order of decreasing Shannon information content for nonscattering clouds. Figure 1 shows the positions of these microwindows relative to molecular emission features. Note that each microwindow contains $\mathrm{CO}_{2}$ lines (for the temperature retrieval, discussed further in Sect. 2.3) whilst avoiding significant contributions from more variable gases such as $\mathrm{H}_{2} \mathrm{O}$.
Table 1. Microwindows for cloud macrophysical parameter retrievals from MIPAS spectra, ordered in terms of priority of selection. Note that the boundaries are multiples of $0.125 \mathrm{~cm}^{-1}$ so are consistent with both the "full-resolution" $\left(0.025 \mathrm{~cm}^{-1}\right.$ grid $)$ and "optimised-resolution" $\left(0.0625 \mathrm{~cm}^{-1}\right.$ grid $)$ spectra.

\begin{tabular}{cc}
\hline MW\# & Wavenumber Range $\left[\mathrm{cm}^{-1}\right]$ \\
\hline 1 & $937.625-940.625$ \\
2 & $941.125-944.125$ \\
3 & $944.500-947.500$ \\
4 & $955.750-958.750$ \\
5 & $948.625-951.125$ \\
6 & $936.000-937.625$ \\
7 & $934.500-935.875$ \\
8 & $953.500-955.000$ \\
9 & $951.875-953.250$ \\
10 & $958.750-960.875$ \\
\hline
\end{tabular}

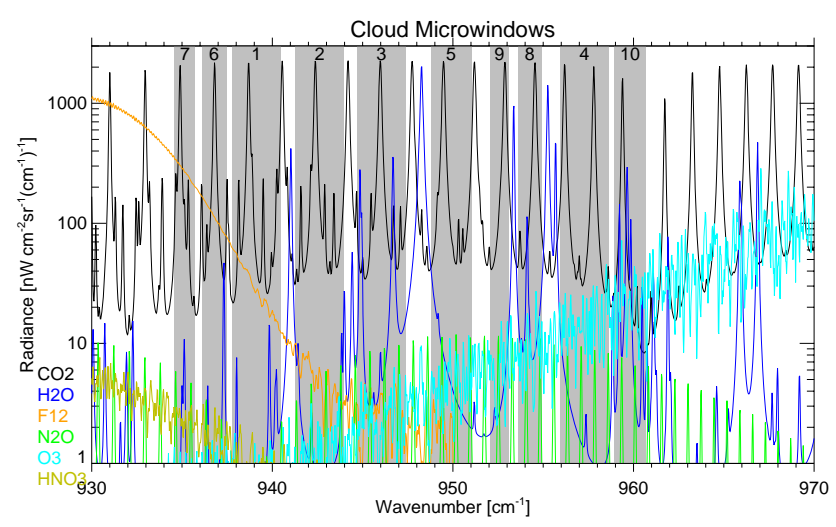

Fig. 1. Modelled full-resolution MIPAS spectrum for a tangent height of $9 \mathrm{~km}$ separated by constituent major emitters, in the spectral region of selected MWs listed in Table 1 - with MW spectral regions shaded.

\subsection{Continuum radiance}

The Reference Forward Model (RFM) (Dudhia, 2005) is a standard radiative transfer model, which has been used here to pre-compute molecular transmittance spectra for each $\mathrm{MW}, \tau_{\nu}$, for each tangent height altitude, based on climatological concentrations (Remedios, 2001). For each MW, it is then possible to identify $n_{\mathrm{MW}}$ spectral points where molecular contributions are expected to be negligible (e.g. where $\left.\tau_{v}>0.95\right)$ using these pre-computed molecular transmittance spectra. It should be noted that at these wavenumbers molecular scattering is also negligible. These $n_{\mathrm{MW}}$ points then form a spectral mask for each MW, from which the continuum radiance, and its associated error, can be calculated. 


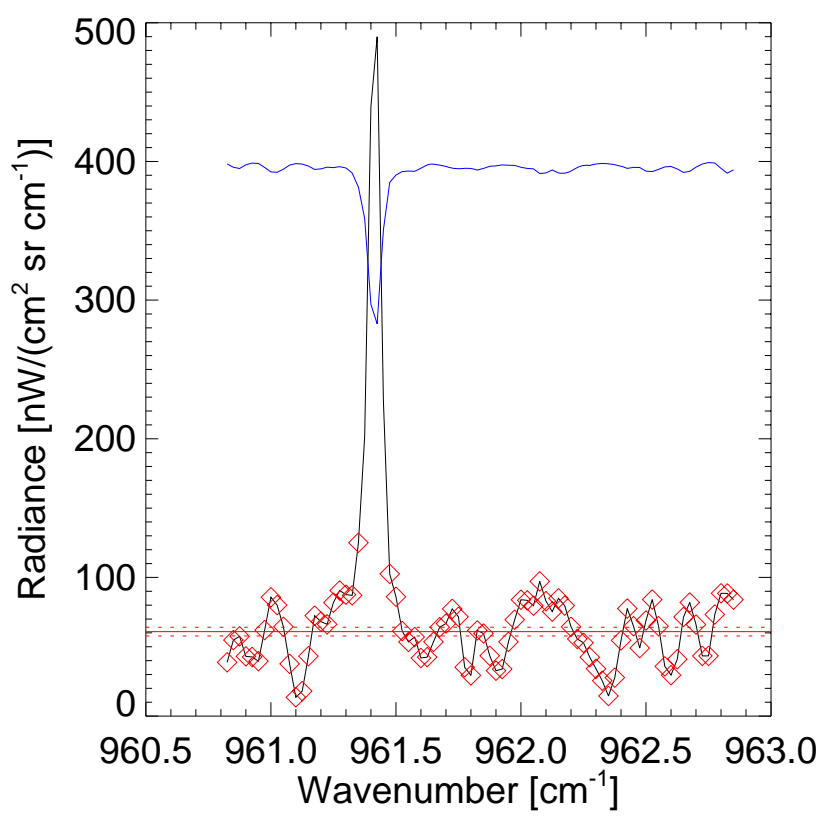

Fig. 2. Illustration of the continuum radiance, showing the RFMsimulated MIPAS radiance at $18 \mathrm{~km}$ (black), the corresponding transmittance spectrum (blue, multiplied by a factor of 400 for visibility), the spectral points utilised to calculate the continuum radiance (red diamonds) along with the calculated continuum radiance (red solid line) for the spectral window considered, with the estimated uncertainty (red dashed lines).

The continuum radiance, $R$, and associated error $\sigma$, can then be established by a simple mean and standard error for each radiance spectrum measured at each tangent height in a MIPAS scan pattern below about $25 \mathrm{~km}$, such that

$R=\frac{1}{n_{\mathrm{MW}}} \sum_{i} L\left(v_{i}, z_{\mathrm{F}}\right)$,

where $L\left(v_{i}, z_{\mathrm{F}}\right)$ is the measured radiance at the $i$ th masked wavenumber $v_{i}$ (in the particular MW) and FOV tangent height $z_{\mathrm{F}}$, and

$\sigma=\frac{1}{n_{\mathrm{MW}}} \sqrt{\sum_{i}\left(L\left(v_{i}, z_{\mathrm{F}}\right)-R\right)^{2}}$,

using standard deviation $D$ such that the standard error is defined as $D / \sqrt{(} n-1)$, where $n_{\mathrm{MW}}$ is the number of points averaged. By assigning an error value based on the actual $D$ rather than the instrument noise, some allowance is made for any residual molecular contributions. Figure 2 illustrates the continuum radiance calculation process.

\subsection{Cloud effective fraction}

The next step is to identify the spectrum containing the cloud-top. One approach could be to use a simple threshold value on the continuum radiance, but since the contin- uum radiance is a strong function of atmospheric temperature and water vapour content as well as cloudiness, finding a suitable threshold value is difficult. The standard MIPAS Cloud Index (CI) method (Spang et al., 2004) attempts to overcome this temperature dependence by taking the ratio of radiance in two spectral regions $\left(792-796 \mathrm{~cm}^{-1}\right.$ and 832 $834 \mathrm{~cm}^{-1}$ ) which react differently to cloud presence. Here, instead, it is preferable to have a scheme dependent upon the continuum radiance within each MW independently, as well as one having a more physical basis, since it parametrises the physical (geometrical and optical) fraction of the FOV filled with cloud. This is done via retrieval of a "Cloud Effective Fraction" (CEF) - a parameter first introduced by Hurley et al. (2009).

The CEF is defined as the fraction of the FOV covered by an optically thick, isothermal cloud with a horizontal cloudtop that would give the same continuum radiance as the observed cloud, assuming both have the same Cloud Top Temperature (CTT). Thus a single parameter $\alpha$ (the CEF), can be used to describe the infinite range possible of cloud extinctions and spatial distributions within the actual FOV (although the concept of a single well-defined CTT in all such cases is questionable). Thus $\alpha$ varies from 0 (cloud-free) to 1 (thick cloud completely filling the FOV) with intermediate values which may correspond either to thick cloud filling a small part of the FOV or thin cloud filling a larger fraction.

Mathematically, $\alpha$ for a FOV having central tangent height $z_{\mathrm{F}}$ is defined as

$\alpha=\frac{\int_{-d}^{z_{\mathrm{c}}-z_{\mathrm{F}}}\left(1-e^{-k_{\mathrm{c}} s(z)}\right) \phi(z) d z}{\int_{-d}^{d} \phi(z) d z}$,

whereby the FOV can be described as extending a distance $2 d$ in the vertical, $z_{\mathrm{c}}$ is the cloud top height measured upward from the Earth's surface, $k_{\mathrm{c}}$ is the cloud extinction coefficient along the limb path $s$, and $\phi(z)$ is the FOV vertical response function. Figure 3 shows the variation of $\alpha$ with extinction and the proportion of the FOV filled with cloud, as CEF is correlated with both. At low extinction (i.e. $k_{\mathrm{c}}<10^{-4} \mathrm{~km}^{-1}$ ), CEF is predominantly determined by extinction, however at the high extinction limit (ie. $k_{\mathrm{c}}>$ $10^{-2} \mathrm{~km}^{-1}$ ), CEF is highly correlated with the proportional filling of the FOV. In fact, a range of extinction and proportional FOV-fillings can combine to produce the same CEF, but outside the optically thin and thick limits, CEF is very much determined by both parameters - and is perhaps best thought of as an optical depth of sorts, normalised to the FOV in question.

From Eq. (3), it follows that, to good approximation,

$\alpha=\frac{R}{B_{\mathrm{c}}}$,

where $R$ is the continuum radiance and $B_{\mathrm{c}}$ is the spectrally averaged Planck function corresponding to the CTT - and this is the definition of CEF used throughout this work. Scattering from cloud particles acts to increase $R$ (as discussed 


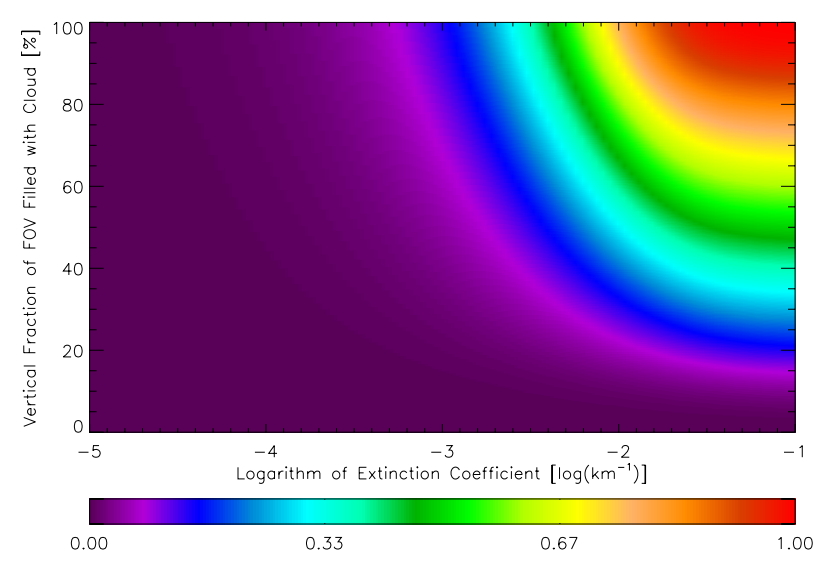

Fig. 3. Variation of CEF with extinction coefficient and the vertical proportion of the MIPAS FOV filled with cloud.

in Sect. 3.3.1) in the infrared, and thus increase the CEF. It is thus possible to obtain, from the Eq. (3) approximation, $\mathrm{CEF}>1$, for scattering clouds. In practice, application to real MIPAS data does not show frequent examples of this, and operationally when this occurs, the CEF is then set to 1 .

This $\alpha$ is then used as the a priori value in the retrieval of the $\mathrm{CEF}$, which is done prior to the full macrophysical retrieval. To retrieve the $\mathrm{CEF}$ from a single microwindow spectrum, it is assumed that the observed radiance can be represented as originating from a homogeneous path with the vertically lower fraction of the FOV $\alpha$ corresponding to an optically thick cloud whilst the upper fraction of the FOV $(1-\alpha)$ originates from molecular emission features above the cloud but at the same local temperature as the cloud-top. Thus, the forward model for the measured spectrally varying radiance $R_{v}$ in order to better estimate the CEF via retrieval, is approximated as

$R_{v}=\alpha B_{\mathrm{c}}+(1-\alpha) B_{\mathrm{c}}\left(1-\tau_{v}\right)$

where $\tau_{v}$ is the same pre-computed (climatological) molecular transmittance used in Sect. 2.2. In practice, this works better if $B_{\mathrm{c}}$ is constrained by a priori information - for instance, by using a temperature climatology.

The CEF is retrieved using an iterative optimal estimation scheme (Rodgers, 2000):

$$
\begin{aligned}
\boldsymbol{x}_{i+1}= & \boldsymbol{x}_{i}+\left(\mathbf{K}_{i}^{T} \mathbf{S}_{\mathbf{y}}^{-1} \mathbf{K}_{i}+\mathbf{S}_{\mathbf{a}}^{-1}\right)^{-1} \\
& \left(\mathbf{K}_{i}^{T} \mathbf{S}_{\mathbf{y}}^{-1}\left(\boldsymbol{y}-\boldsymbol{f}_{i}\right)-\mathbf{S}_{\mathbf{a}}^{-1}\left(\boldsymbol{x}_{i}-\boldsymbol{a}\right)\right),
\end{aligned}
$$

where subscript $i$ denotes the iteration number, the state vector $\boldsymbol{x}$ contains the parameters to be retrieved, the measurement vector $\boldsymbol{y}$ contains the measurements, $\boldsymbol{f}$ is the forward model (Sect. 2.5) applied to the current iteration of $\boldsymbol{x}, \mathbf{K}$ is the Jacobian matrix containing elements $\partial \boldsymbol{f} / \partial \boldsymbol{x}, \mathbf{S}_{\mathbf{y}}$ is the error covariance matrix of $\boldsymbol{y}, \boldsymbol{a}$ is the a priori estimate of $\boldsymbol{x}$ and $\mathbf{S}_{\mathbf{a}}$ is the error covariance of $\boldsymbol{a}$.
In the case of the CEF retrieval, the state vector contains the CEF and a retrieved value of the Planck function, and the a priori vector contains the estimated $\mathrm{CEF} \alpha$, as calculated from the continuum radiance, and the Planck function evaluated at the climatological for that tangent height. The measurement vector contains the spectrally varying radiance $L_{v}$, which the forward model $R_{v}$ seeks to reproduce given the appropriate $\alpha$ and $B_{\mathrm{c}}$.

Error on the measured spectrum is accounted for in the error covariance matrix

$\mathbf{S}_{\mathbf{y}}=\sigma_{n}^{2} \mathbf{I}_{\mathbf{n}}$

for $\sigma_{n}=$ NESR (the Noise Equivalent Spectral Radiance, which for the case at hand is roughly $50 \mathrm{nW}$ ) and the $n \times n$ identity matrix $\mathbf{I} \mathbf{n}$. The uncertainty in the a priori is accounted for in the a priori covariance matrix $\mathbf{S}_{\mathbf{a}}$, such that

$\mathbf{S}_{\mathbf{a}}=\left(\begin{array}{cc}\sigma_{\alpha}^{2} & 0 \\ 0 & \sigma_{B_{\mathrm{c}}}^{2}\end{array}\right)$,

taking the uncertainty in the estimated CEF to be $\sigma_{\alpha}^{2}=$ $(1.0)^{2}$. The uncertainty in the blackbody radiance evaluated at the tangent height can be obtained from the expression

$\sigma_{B_{\mathrm{c}}}^{2}=\left(\left.\frac{\partial B}{\partial T}\right|_{\bar{\nu}, T_{\mathrm{F}}}\right)^{2} \sigma_{T_{\mathrm{F}}}^{2}$,

where $\sigma_{T_{\mathrm{F}}}^{2}$ is the variance in the climatological temperature at $z_{\mathrm{F}}$, typically taken around $20 \mathrm{~K}$ to reflect the range of temperatures expected throughout the range of altitudes spanned by the FOV.

The retrieval error, stemming from the retrieval process itself, given the uncertainties caused by noise on the measured spectra (in $\mathbf{S}_{\mathbf{y}}$ ), as well as uncertainties in the assumed a priori (in $\mathbf{S}_{\mathbf{a}}$ ), is given by

$\mathbf{S}_{\mathbf{X}}=\left(\mathbf{K}^{T} \mathbf{S}_{\mathbf{y}}^{-1} \mathbf{K}+\mathbf{S}_{\mathbf{a}}^{-1}\right)^{-1}$

Once this retrieval for CEF has converged, the cloud-top is identified as lying in the highest altitude spectrum where the retrieved $\alpha>0.1$. Finally, the retrieved value of $\alpha$ is also used as a "measurement" in the macrophysical parameter retrieval itself (Sect. 2.4). In principle, Eq. (5) also yields an "improved" estimate of $B_{\mathrm{c}}$ but, given the crudeness of this approximation, it is preferred to re-use the original climatological temperature profile.

\subsection{Macrophysical parameter retrieval}

The macrophysical parameters are retrieved using the optimal estimation formulation given in Eq. (6). 


\subsubsection{State vector}

The state vector $\boldsymbol{x}$ contains the parameters to be retrieved, and in this case is defined as

$\boldsymbol{x} \equiv\left(\begin{array}{c}z_{\mathrm{c}} \\ B_{\mathrm{c}} \\ \mu_{\mathrm{c}}\end{array}\right)$,

where $z_{\mathrm{c}}$ is the cloud-top height $(\mathrm{CTH}), B_{\mathrm{c}}$ is the Planck function evaluated at the cloud-top temperature $T_{\mathrm{c}}$ (CTT) at the mid-point of the microwindow, and the logarithm of the extinction coefficient (CEX) is defined as

$\mu_{\mathrm{c}}=\log _{10} k_{\mathrm{c}}$,

where $k_{\mathrm{c}}$ is the extinction coefficient (in $\mathrm{km}^{-1}$ ) measured along the limb line-of-sight. $k_{\mathrm{c}}$ varies spectrally, and so the $\mu_{\mathrm{c}}$ retrieved in each MW corresponds to the average extinction in that MW - and any subsequent combination of MW results should keep this spectral variance in mind, even though it will be small in such a short spectral range.

In practice, $k_{\mathrm{c}}$ is the extinction coefficient corresponding to the total extinction along the MIPAS limb path, including contributions from both atmospheric and cloud components of measured signal. However, as discussed in Sect. 2.1, the MWs in which the cloud properties are derived have been pre-selected such that the atmospheric contributions will be negligible in comparison with the cloud signal, having transmittance greater than $95 \%$. Thus, to good approximation, the retrieved value of $k_{\mathrm{c}}$ will correspond to the extinction of the cloud along the MIPAS limb path.

\subsubsection{Measurement vector}

The vector $\boldsymbol{y}$, containing the measurements used for the retrieval, is defined as

$\boldsymbol{y} \equiv\left(\begin{array}{c}R_{\mathrm{u}} \\ R_{\mathrm{c}} \\ R_{\mathrm{l}} \\ \alpha\end{array}\right)$,

where $R_{\mathrm{c}}$ is the continuum radiance (Sect. 2.2) from the FOV containing the cloud-top, having the retrieved cloud effective fraction $\alpha$, while $R_{\mathrm{u}}$ and $R_{\mathrm{l}}$ are the continuum radiances from the FOVs immediately above and below, calculated in the same manner. The measurement covariance matrix $\mathbf{S}_{\mathbf{y}}$ is diagonal, with variances given by the errors from the continuum radiance and CEF retrieval. Although $R_{\mathrm{c}}$ and $\alpha$ are derived from the same spectrum, the argument is that $\alpha$ depends on the spectral structure whereas $R_{\mathrm{c}}$ is derived from the spectrally flat regions - and hence the two may be regarded as independent.

The radiance $R_{\mathrm{u}}$ from the FOV above the cloud-top is expected to have a value $\sim 0$ (since the CEF for this FOV will have been retrieved with a value $<0.1$, Sect. 2.3) and serves simply to constrain the retrieval from placing the cloud-top too high. The inclusion of the CEF in the measurement vector is discussed in the next section.

\subsubsection{A priori information}

This scheme essentially attempts to retrieve three macrophysical parameters from two non-zero continuum measurements, $R_{\mathrm{c}}$ and $R_{\mathrm{l}}$. The usual method for dealing with such under-determined problems is to supply independent a priori information. Due to the spatial inhomogeneity of cloud structures, obtaining useful direct a priori information on any of the three retrieved parameters is impractical - however, there are indirect a priori constraints on the relationships between the retrieved parameters.

The first a priori constraint is represented by the CEF ( $\alpha$ in Eq. 13) and is more conveniently introduced into the measurement vector $(\boldsymbol{y})$ itself rather than in the conventional a priori vector $\boldsymbol{a}$. This acts as a constraint on the CTH and CEX values, as described in Sect. 2.3.

A second source of a priori information is the background temperature profile obtained, for example, from climatology or meteorological analysis fields. Assuming this is not significantly perturbed in the presence of clouds, this acts as a constraint on the CTH and CTT, since the cloud-top temperature would be expected to correspond to a point on this profile.

Having identified the spectrum containing the cloud-top, the a priori estimate for the cloud-top height is set as the nominal tangent height for that measurement $z_{\mathrm{F}}$, and its corresponding uncertainty $\sigma_{\mathrm{za}}$ set to $\pm 1 \mathrm{~km}$. This corresponds to the range of the effective FOV width $\sim \pm 1.5 \mathrm{~km}$, since it is reasonable that this should envelope the uncertainty in cloud top height, if the cloud detection method is trustworthy.

For this altitude, the background temperature profile provides an equivalent radiance $B_{\mathrm{F}}$, and uncertainty $\sigma_{\mathrm{BF}}$ which is typically equivalent to a temperature uncertainty of $\pm 10 \mathrm{~K}$. However, uncertainty with which $z_{\mathrm{F}}$ represents the actual cloud-top height, and the variation of radiance with altitude $b=d B / d z$ (see Eq. 15) also have to be taken into account when calculating the a priori covariance matrix elements.

There is no reasonable a priori estimate for optical thickness so it is just set at a typical mid-range value (e.g. $\left.\mu_{\mathrm{a}}=-2.5\right)$ with a large uncertainty $\sigma_{\mu_{a}}= \pm 0.5$, to capture the range of extinction for which the cloud forward model (Sect. 2.5) is applicable.

Thus the a priori vector is given by

$\boldsymbol{a}=\left(\begin{array}{c}z_{\mathrm{F}} \\ B_{\mathrm{F}} \\ \mu_{\mathrm{a}}\end{array}\right)$.

In addition, it is assumed that the Planck function (evaluated at the spectral mid-point of the microwindow in question) varies linearly with altitude within the cloud with a known gradient, such that

$B(z)=B_{\mathrm{c}}+b\left(z-z_{\mathrm{c}}\right)$, 
where $B_{\mathrm{c}} \equiv B\left(T_{\mathrm{c}}\right)$ is the Planck function for the cloud top temperature, and $b=d B / d z$ is the vertical gradient derived from an external (e.g. climatological) estimate of the background atmospheric temperature profile. This vertical gradient $b<0$ in the troposphere and $b>0$ in the stratosphere, and is the radiance equivalent to the temperature lapse rate.

Assuming that the Planck function varies linearly with altitude (Eq. 15) in order to estimate the uncertainty on the cloud top radiance, the a priori covariance matrix is given by

$\mathbf{S}_{\mathbf{a}}=\left(\begin{array}{ccc}\sigma_{\mathrm{za}}^{2} & b^{2} \sigma_{\mathrm{za}}^{2} & 0 \\ b^{2} \sigma_{\mathrm{za}}^{2} & \left(\sigma_{\mathrm{BF}}^{2}+b^{2} \sigma_{\mathrm{za}}^{2}\right) & 0 \\ 0 & 0 & \sigma_{\mu_{a}}^{2}\end{array}\right)$.

\subsection{Cloud forward model}

The essential assumption within the macrophysical retrieval scheme is that a cloud can be represented as a homogeneous "grey" absorber characterised by the retrieved parameters. The cloud forward model (CFM) $\boldsymbol{f}$ calculates the continuum radiance originating from a cloud described by $z_{\mathrm{c}}, T_{\mathrm{c}}$ and $k_{\mathrm{c}}$, and assumes that there is no spectral variation in absorption or in the Planck function over the limited spectral width of each microwindow.

\subsubsection{Pencil-beams}

The continuum radiance $L_{\mathrm{t}}$ of an infinitesimal solid-angle pencil-beam viewing at tangent height $z_{\mathrm{t}}$ within the cloud (i.e. $z_{\mathrm{t}}<z_{\mathrm{c}}$ ) is given by the standard radiative transfer equation for local thermodynamic equilibrium, assuming no molecular contributions from the atmosphere itself, and no scattering:

$L_{\mathrm{t}}=\int_{s} B(s) \frac{d \tau}{d s} d s$,

where $B(s)$ is the Planck function evaluated at the spectral mid-point of the microwindow along the path $s$, and $\tau(s)$ is the transmittance along the path $s$, given by

$\tau=\exp \left(-k_{\mathrm{c}} s\right)$

Using simple circular geometry (ignoring refraction and assuming the Earth's radius, $r_{\mathrm{e}} \gg z$ ), the path distance and altitude relative to the tangent point values are related by

$\left(s-s_{\mathrm{t}}\right)^{2} \simeq 2 r_{\mathrm{e}}\left(z-z_{\mathrm{t}}\right)$.

Eq. (17) can then be solved to give

$L_{\mathrm{t}}=\left(B_{\mathrm{c}}+\frac{b}{r_{\mathrm{e}} k_{\mathrm{c}}^{2}}\right)(1-\tau)-\left(\frac{b s}{2 r_{\mathrm{e}} k_{\mathrm{c}}}\right)(1+\tau)$.

The appearance of the retrieved parameter $k_{\mathrm{c}}$ in the denominator makes this potentially numerically unstable in the optically-thin limit, so a more computationally robust approximation is preferred, such that

$L_{\mathrm{t}} \simeq\left(B_{\mathrm{c}}+\frac{2}{3} b\left(z_{\mathrm{t}}-z_{\mathrm{c}}\right) \tau\right)(1-\tau)$, which agrees with the exact solution in the asymptotic limits of transmittance. In the optically thick limit $(\tau=0)$ cloud effectively just emits from its upper surface and $L_{\mathrm{t}} \rightarrow$ $B_{\mathrm{c}}$, as expected. In the optically thin limit $(\tau \rightarrow 1)$, the emission effectively comes from the point one third of the vertical distance from the tangent point to the cloud-top, $L_{\mathrm{t}} \rightarrow\left(\frac{1}{3} B_{\mathrm{c}}+\frac{2}{3} B_{\mathrm{t}}\right)(1-\tau)$, where $B_{\mathrm{t}} \equiv B\left(z_{\mathrm{t}}\right)$ from Eq. (15).

\subsubsection{FOV convolution}

The MIPAS FOV response function is represented by a vertical trapezium with a $4 \mathrm{~km}$ base and a $2.8 \mathrm{~km}$ top when projected onto the atmospheric limb. With tangent heights spaced at $3 \mathrm{~km}$ intervals for the original full-resolution measurements, this gives a small overlap between adjacent measurements, but a much larger overlap for the $1.5 \mathrm{~km}$ spacing used in the "optimised-resolution" measurements employed since 2005.

This FOV function $\phi$ is sampled at $N$ points (in practice, $N=9$ ), which determine the altitudes $z_{j}$ for which the pencil-beam calculations are performed. The measured continuum radiance is then represented by a numerical convolution of the pencil-beam radiances at these altitudes $\left(L_{\mathrm{t}_{j}}\right)$, such that

$R=\sum_{j=1}^{N} a_{j} L_{\mathrm{t}_{j}}$,

where $a_{j}$ are coefficients of the normalised FOV convolution function at each pencil-beam altitude $z_{j}$ multiplied by the "infinitestimal" integration step. Below the cloud-top, as the integration occurs at these finite points, the radiance is assumed to vary linearly between any two integration points - but at the cloud-top, there is a step function in radiance between that emitted by the cloud, and that emitted by the clear atmosphere.

\subsubsection{Cloud effective fraction}

As mentioned in Sect. 2.4.2, the CEF defined in Eq. (4) is included in the measurement vector, therefore has to be evaluated by the forward model. Using Eq. (22)

$\alpha=\frac{\sum_{j=1}^{N} a_{j} L_{t_{j}}}{B_{\mathrm{c}}}$.

In the optically-thick limit, $L_{\mathrm{t}} \sim B_{\mathrm{c}}$ (Eq. 21) for pencilbeams which intersect the cloud, whilst $L_{\mathrm{t}}=0$ for pencilbeams above the cloud top. Thus, this expression for $\alpha$ for a thick cloud effectively is dependent only upon the relative geometric filling of the FOV. 


\subsubsection{Definition of cloud forward model}

Thus, the CFM $f$ is simply Eq. (22) applied to each of the FOVs available in the measurement vector $\boldsymbol{y}$, along with the definition of the CEF, $\alpha$, given in Eq. (23). Furthermore, since these are analytic expressions, analytic derivatives are used to calculate elements of the Jacobian matrix $\mathbf{K}$.

\subsection{Combining microwindow results}

\subsubsection{Statistical combination}

Retrievals $\boldsymbol{x}_{k}$, and associated covariances $\mathbf{S}_{\mathbf{x} k}$, are obtained from each of the $M=10$ microwindows. These results can then be combined using the standard statistical procedure for independent estimates, such that

$$
\begin{aligned}
\hat{\mathbf{S}}_{\mathbf{x}}^{-1} & =\sum_{k=1}^{M}\left(\mathbf{S}_{\mathbf{x} k}\right)^{-1} \text { and } \\
\hat{\boldsymbol{x}} & =\hat{\mathbf{S}}_{\mathbf{x}} \sum_{k=1}^{M}\left(\mathbf{S}_{\mathbf{x} k}\right)^{-1} \mathbf{x}_{k},
\end{aligned}
$$

where $\hat{\boldsymbol{x}}$ and $\hat{\mathbf{S}}_{\mathbf{x}}$ represent the combined estimate and its covariance. There is an assumption here that the retrieved parameters do not vary spectrally - at least across the tens of wavenumbers represented by the selected microwindows (cloud-top radiances are converted to cloud-top temperatures prior to the combination). Extinction, of course, does vary spectrally - however over the small spectral range sampled by the MWs, this variation is also small. It also ignores the fact that the same a priori temperature climatology is used for each estimate, so the separate microwindow results are not strictly independent.

\subsubsection{Spike tests}

This combination step also allows a spike-test to be applied - that is, a removal of results from any microwindows which deviate significantly from the mean. The $\chi^{2}$ statistic is computed for each microwindow individually

$\chi_{k}^{2}=\left(\mathbf{x}_{k}-\hat{\mathbf{x}}\right)^{T} \hat{\mathbf{S}}_{\mathbf{x}}^{-1}\left(\mathbf{x}_{k}-\hat{\mathbf{x}}\right)$,

and if the microwindow with the highest $\chi^{2}$ value exceeds the average $\chi^{2}$ by some factor (e.g. 2) its results are removed from the combination and the test repeated for the remaining microwindows.

\subsubsection{Error inflation}

In theory, the covariance $\hat{\mathbf{S}}_{\mathbf{x}}$ should contain the random error information on the retrieved values. However, it is recognised that this is an optimistic assumption since it makes no allowance for the forward model errors or approximations, which have systematic components. If the different microwindows produce a large scatter of results, then the standard deviation $D$ of this distribution is likely to be a better estimate of the actual uncertainty, although this does not necessarily allow for forward model errors (see Sect. 3) either since all microwindows make the same assumptions. A three-element vector of scale-factors $\boldsymbol{e}$ is constructed to take the maximum of these to conservatively estimate the largest error likely to propagate through from the individual retrievals, such that

$e_{m}=\max \left(1, \frac{\mathrm{D}_{m}}{\sigma_{m}}\right)$

where $\sigma_{m}$ is the square root of the $m$ th diagonal element in the matrix $\hat{\mathbf{S}}_{\mathbf{X}}$ (i.e. the uncertainty in parameter $x_{m}$ according to the covariance matrix) and $D_{m}$ is the actual standard deviation of the parameter $x_{m}$ from the different microwindow results.

The retrieval covariance is then "inflated" to produce the final covariance, such that

$\hat{\mathbf{S}}_{\mathbf{x} m \mathrm{~m}}^{\prime}=e_{m}^{2} \hat{\mathbf{S}}_{\mathbf{x} m \mathrm{~m}}$.

\subsection{Operational considerations}

In an operational processor, it is desirable to have alternative schemes available to perhaps retrieve fewer parameters in situations where the full retrieval fails due to an insufficient number of microwindows providing retrievals which converge or pass the spike test, or if insufficient measurements are available, which happens most commonly when the cloud-top is detected in the lowest spectrum in the limb scan.

Assuming that a cloud-top has been detected somewhere in the scan, the operational algorithm attempts the following retrieval schemes in sequence until one returns valid results for at least three microwindows.

1. If available, using the measurement from the tangent height below the cloud-top $R_{1}$ (i.e. the cloud-top not located in the lowest tangent height in the scan), with a priori extinction information given by $\mu_{\mathrm{a}}=-2.5$ (i.e. mid-range value). This is the full three parameter retrieval $\left(z_{\mathrm{c}}, T_{\mathrm{c}}, \mu_{\mathrm{c}}\right)$ from three measurements $\left(R_{\mathrm{c}}, R_{1}, \alpha\right)$ plus the nominally zero radiance measurement $R_{\mathrm{u}}$ from the tangent height above the cloud-top, as described previously.

2. As (1) but setting $\mu_{\mathrm{a}}=-1.0$, giving a "thick cloud" assumption $\left(k_{\mathrm{c}}=0.1 \mathrm{~km}^{-1}\right)$. Such a large initial guess value of extinction reduces the Jacobians with respect to this parameter to nearly zero, effectively leaving just two parameters $\left(z_{\mathrm{c}}, T_{\mathrm{c}}\right)$ to be retrieved from three measurements $\left(R_{\mathrm{c}}, R_{1}, \alpha\right)$.

3. As (2) but without $R_{1}$ - that is, the "thick cloud" assumption allowing for retrieval of two parameters 
$\left(z_{\mathrm{c}}, T_{\mathrm{c}}\right)$ from only one tangent height using two measurements $\left(R_{\mathrm{c}}, \alpha\right)$. This relies on the CEF retrieval in order to separate the two parameters.

\section{Assessment of errors stemming from model assumptions}

\subsection{Retrieval error and real error}

It must be noted that errors reported by the retrieval error covariance matrix $\mathbf{S}_{\mathbf{X}}$ describe only the errors expected due to the statistical nature of an optimal estimations retrieval process, and include only estimates of the uncertainties introduced into the retrieval by noise on the measurement, and by choise of the a priori estimates, as discussed in Rodgers (2000). $\mathbf{S}_{\mathbf{X}}$ can be used to judge the convergence of a retrieval. However, it in no way encompasses the real error stemming from inaccuracies and insufficiencies in the forward model itself unless the forward model is physically complete. In this section, a thorough assessment of shortcomings of the forward model - and the errors that are thus introduced - is carried out. As well, an assessment is carried out on how other pertinent atmospheric and instrument uncertainties can further add to the overall real error in retrieved values.

Insufficiencies in the forward model are the dominant error sources, and errors propagating from such are often much larger than the retrieval error itself. As in any model, there are limits to the applicability of this algorithm. However, as long as the limits of applicability of this model, and the errors implicit because of the basic assumptions, are well known, it can be used within the discussed range of confidence.

\subsection{Limitations of the cloud forward model: extinction range of sensitivity}

It is worth considering the optical thickness range over which the forward model is applicable. Consider first an optically thin cloud which completely fills the FOV. From the CFM, it follows that the total radiance in the FOV is

$R_{\mathrm{c}}=B_{\mathrm{c}}\left(1-e^{-k_{\mathrm{c}} s}\right) \simeq B_{\mathrm{c}} k_{\mathrm{c}} s$.

The CEF of this thin cloud is

$\alpha=\frac{R_{\mathrm{c}}}{B_{\mathrm{c}}} \simeq k_{\mathrm{c}} s$.

Assuming a pathlength of approximately $300 \mathrm{~km}$, and that clouds are detected only for $\alpha>0.1$, this implies that the thinnest cloud which can be registered using this detection method has an extinction coefficient of $0.0003 \mathrm{~km}^{-1}$. Furthermore, for clouds having low optical depths (and in particular, those having a vertically thin distribution of cloud particles), scattering becomes a non-negligible process, and the CFM is not sufficient to describe the emitted radiance.
However, such thin clouds are unlikely to be homogeneous in the MIPAS FOV, so even if scattering were included in the retrieval model, it is unlikely to give a significantly improved retrieval of the actual cloud properties. It should be noted, however, that the exclusion of scattering from the model thus introduces a systematic offset to the retrieved parameters as scattering generally acts to increase the radiance at these wavelengths.

Turning to the optically thick limit, assume that the extinction is indistinguishable from infinity for path transmittances less than $1 \%$ :

$\tau=e^{-k_{\mathrm{c}} s}=0.01$.

Given an estimated pathlength of $300 \mathrm{~km}$, this yields that clouds with $k_{\mathrm{c}}>0.015 \mathrm{~km}^{-1}$ are indistinguishable from one another.

Therefore, it is reasonable to expect that extinction can be retrieved in the approximate range of $-4 \leq \mu_{\mathrm{c}} \leq-2$.

\subsection{Limitations of cloud forward model: scattering}

The process of scattering tends to increase the radiance emitted by a cloud. In the range of extinction coefficients between $10^{-4}-10^{-2} \mathrm{~km}^{-1}$, this is less than by a factor of two or three (see Sect. 3.3.1). This effect is accounted for in terms of forward modelling error in the retrieval process, but should be realised as a limitation of the model. The inclusion of scattering into this algorithm would imply that it could not be used operationally, as addition of scattering into any calculation increases the computational cost of the problem dramatically. It also introduces extra uncertain cloud parameters to the problem, such as scattering particle characteristics, which is out of the scope of this work. In the "MIPclouds" project, microphysical cloud parameters are addressed in a separate microphysical cloud parameter retrieval (Spang et al., 2008).

\subsubsection{Validation using KOPRA simulations}

Whilst the forward model (CFM) discussed in the past few sections well describes an optically grey cloud, it is not necessarily a good representation of real clouds, which scatter radiation in and out of the line-of-sight. It is a useful exercise to compare the CFM with a more realistic model, which allows for scattering - and then to see how well the current retrieval is able to accurately retrieve the macroscopic parameters of a more realistic cloud.

To this end, the Karlsruhe Optimised and Precise Radiative transfer Algorithm (KOPRA) is introduced to provide more accurate simulations of scattering clouds, using a layer-by-layer approach of homogeneous layers in which the radiative transfer proceeds through a succession of extinctions, emissions and scatterings, as described in Höpfner and Emde (2005). KOPRA has been used in the European Space Agency "Cloud Information Retrieval from MIPAS Measurements" "MIPclouds" study (Spang et al., 2008) to create a cloud spectral database for Polar Stratospheric Clouds, 

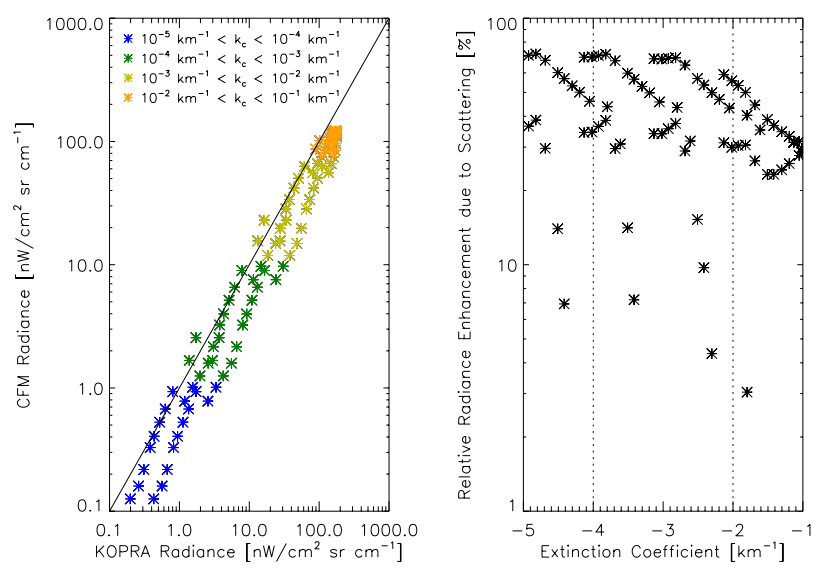

Fig. 4. Comparison of KOPRA (scattering and thus more realistic) and CFM (non-scattering) radiances, as a function of extinction coefficient, as indicated by colour-scale (left panel). Relative radiance enhancement due to scattering is plotted on the right, with the range of extinction sensitivity in the CFM noted by dashed lines.

cirrus and liquid water clouds for a wide range of macroand micro-physical cloud parameters, including atmospheric contributions as well as those resulting from the cloud presence itself.

For the purposes of this exercise, mid-latitude cirrus cases from the database will be considered, as they form a typical and frequent class of high clouds detected by MIPAS. Midlatitudinal cirrus has been modelled here as having cloud top heights between $6.5 \mathrm{~km}$ and $12.5 \mathrm{~km}$, cloud depths between $0.5 \mathrm{~km}$ and $4 \mathrm{~km}$, effective radii between $4.0 \mu \mathrm{m}$ and $90.0 \mu \mathrm{m}$, volume densities between $1.1 \mathrm{~m}^{-3}$ and $1.1 \times 10^{7} \mathrm{~m}^{-3}$, and ice water content between $10.0^{-6} \mathrm{~g} \mathrm{~m}^{-3}$ and $1.0 \mathrm{~g} \mathrm{~m}^{-3}$, with microphysical parameters defined by Baran (2001). This results in clouds modelled with extinction coefficients between approximately $10^{-5} \mathrm{~km}^{-1}$ and $10^{2} \mathrm{~km}^{-1}$, although only cloud having CEX $<-1$ is considered here.

For the sake of argument, only KOPRA simulations with cloud top heights of $10.5 \mathrm{~km}$ and $11.5 \mathrm{~km}$ and cloud depths of $4.0 \mathrm{~km}$ are considered, even though for the $11.5 \mathrm{~km}$ case the lower FOV will not have the bottom $500 \mathrm{~m}$ cloud-filled as this represents a negligible radiance discrepancy. Figure 4 compares the radiances coming from KOPRA-simulated clouds and those calculated by the CFM presented here, for the considered cases. The different cases are colour-coded by extinction coefficient.

Given that the CFM seems able to represent singlescattering clouds (as modelled by KOPRA) to within an order of magnitude, it is interesting to see how well the macroscopic retrieval can estimate the retrieved parameters, applying the full three-parameter retrieval. Since KOPRA is a physically more rigorous model, this should give a metric of the skill with which the retrieval can determine cloud parameters for real clouds of various optical thicknesses.
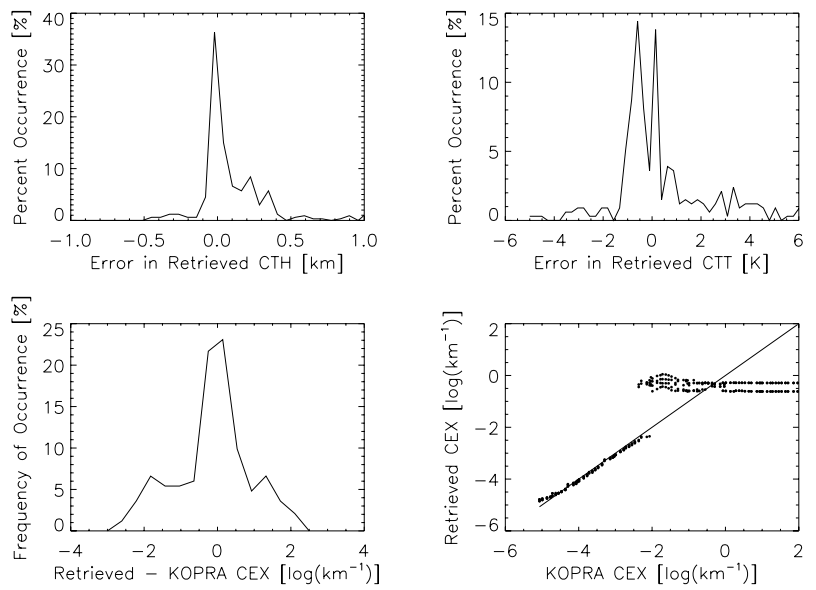

Fig. 5. Retrieved CTH, CTT and CEX for all KOPRA-simulated clouds having CTHs of $10.5-11.5 \mathrm{~km}$, including those which are outside the purported range of applicability of the model. Top panels: probability distribution functions of difference between retrieved and simulated CTH (left) and CTT (right) for KOPRAsimulated clouds. Lower left panel: probability distribution function of the difference between KOPRA-simulated CEX and the retrieved CEX. Lower right panel: scatterplot of retrieved CEX (right panel) for KOPRA-simulated clouds. Black line shows one-to-one division.

Again, considering the mid-latitudinal cirrus spectra used in the "MIPclouds" study, the macroscopic retrieval has been run to this end, the results of which are shown in Fig. 5.

It appears that the retrieval does a fairly consistent job of determining extinction, in the sense that it is able to differentiate between thin and thick cloud. For lower extinction values $\left(<10^{-2} \mathrm{~km}^{-1}\right)$, the retrieval is able to make a reasonable estimate of the extinction, certainly retrieving CEX within $0.5 \log \left(\mathrm{km}^{-1}\right)$. However, whilst the retrieval recognises cases of higher extinction as such, it does not usually get the extinction coefficient quite right for high cloud extinction (see bottom right panel in Fig. 5), since the emitted radiance saturates toward the opaque limit, from values upwards of $10^{-2}-10^{-1} \mathrm{~km}^{-1}$. It is these thick examples which cause the large error range in the lower left panel of Fig. 5.

In terms of the retrieved cloud top heights and cloud top temperatures, the retrieval tends to retrieve most cases to within $50 \mathrm{~m}$ and $1 \mathrm{~K}$, although cases of higher error exist, up to $\approx 500 \mathrm{~m}$ and $\approx 5 \mathrm{~K}$. Such instances of larger error generally correspond to cases of high cloud effective fraction (extinctions greater than about $0.1 \mathrm{~km}^{-1}$ ) when the retrieval tends to overestimate cloud top height and temperature, and to accordingly underestimate the extinction.

In conclusion, retrievals of KOPRA simulations (which are expected to better represent true clouds as they scatter radiance) using the simple CFM are usually reliable to within $50 \mathrm{~m}, 1 \mathrm{~K}$ and half an order of magnitude of the extinction coefficient, for the extinction range for which this CFM is 
asserted to be representative. These errors are not all random - rather the non-scattering CFM will systematically underestimate the true radiance in the infrared which is measured due to scattering for a given set of macro- and micro-physical parameters by up to a factor of two or three, which will effect the retrieved parameters. Thus, the CFM and retrieval based upon it work reliably within the design bounds and estimated retrieval errors provided by the error covariance matrix $\mathbf{S}_{\mathbf{x}}$, well representing clouds for which scattering is not the dominant radiative process, and for which the assumption of homogeneity is satisfied. As such, these error values do not represent the real range of errors expected when the algorithm is applied to real data, which is dominated by errors due to inhomogeneities in the cloud fields.

\subsection{Limitations of cloud forward model: homogeneity}

As a basic assumption of the CFM, the modelled cloud is assumed to fully-fill the horizontal domain of the FOV and to extend downwards to the surface of the Earth from the modelled cloud top height. Horizontal continuity across regions as big as the FOV is a realistic assumption for cirrus fields, which can extend in sheets for several hundreds of kilometres, although potentially not for individual clouds or lower cloud layers. Furthermore, obviously no cloud will actually extend vertically in such a manner - this assumption is simply taken so that the cloud fills the modelled FOV to the bottom of the FOV below that in which the cloud top is identified. Since the FOV integration does not consider any pencil-beam radiance contributions beyond this, the effective cloud base is that of the lowest extent of that FOV. These assumptions have implications upon the retrieved parameters:

1. Optically-thin clouds contain good information on all three macrophysical cloud parameters discussed here but particularly on CEX. However, in this case there is some sensitivity to the FOV-filling assumptions.

- Horizontal filling assumption: if, in reality, the cloud does not fully fill the horizontal (both across FOV, or along the FOV) extent of the FOV as assumed, the retrieved CEX will be less than the real cloud extinction value. Without further information (for example, imaging to show the horizontal extent of the cloud with respect to the measurement FOV), this remains an intractable problem.

- Vertical filling assumption: similarly, if the cloud does not extend vertically to the bottom of the lowest FOV considered in the CFM, a similar effect will be noticed. However, this effect should be minimised because at these wavelengths most clouds should be opaque to radiation higher than the cloud base, for the extinction range of applicability.
2. Optically-thick clouds will have good information on cloud top height and temperature, but generally will not be sensitive to variations in extinction. Assumptions on the relative filling of the FOV will thus affect the retrieved values of CTH and CTT, and the value of CEX will be fairly arbitrary, having a value reflecting a opaque or near-opaque cloud. Unless the cloud becomes opaque below the cloud base is reached there will be a significant discrepancy in radiance measured in comparison to that expected by the CFM - and hence the CTHs should be underestimated.

Inhomogeneities are expected to be the sources of the dominant error in the retrieval, even though the magnitudes of such errors are not accounted for in any of the retrieval error estimates.

\subsubsection{Using the CFM}

Although not accurately representative for real scattering clouds, the clearest way to see the manner in which the retrieval algorithm responds to clouds not satisfying the homogeneity assumptions is to provide simulations using the CFM.

\section{Case 1: horizontal inhomogeneity}

A typical example of horizontal inhomogeneity is a cloud field filling the across-FOV horizontal extent of the FOV, which has an inconstant cloud top, or a inconstant extinction - or a combination of the two. A simple test of the behaviour of the retrieval algorithm is thus to model the FOV as fullyfilled (up to the local CTHs) with two different clouds, each having a different CTH/CTT and CEX.

The FOV is considered to have two distinct clouds, which can be characterised by cloud top heights $\mathrm{CTH}_{1}$ and $\mathrm{CTH}_{2}$, corresponding cloud top temperatures $\mathrm{CTT}_{1}$ and $\mathrm{CTT}_{2}$, having extinctions $\mathrm{CEX}_{1}$ and $\mathrm{CEX}_{2}$, which fill fractions $p_{1}$ and $p_{2}$ of the FOV, respectively, such that $p_{1}+p_{2}=1$. The CFM is used to simulate the radiance each cloud would emit if it were to homogeneously fill the full FOV up to its CTH ( $L_{1}$ and $L_{2}$, respectively), and then the two are combined to simulate the radiance emitted in the inhomogeneously-filled FOV $L_{\text {in }}$, with each of them filling a particular fraction:

$L_{\text {in }}=p_{1} L_{1}+p_{2} L_{2}$.

The effective cloud top height $\left(\mathrm{CTH}_{\mathrm{in}}\right)$, cloud top temperature $\left(\mathrm{CTT}_{\mathrm{in}}\right)$, and logarithm of cloud extinction along the limb path $\left(\mathrm{CEX}_{\mathrm{in}}\right)$ of the overall inhomogeneous FOV are defined as the fraction-weighted averages of the two sets of cloud parameters:

$$
\begin{aligned}
& \mathrm{CTH}_{\text {in }}=p_{1} \mathrm{CTH}_{1}+p_{2} \mathrm{CTH}_{2} \\
& \mathrm{CTT}_{\text {in }}=p_{1} \mathrm{CTT}_{1}+p_{2} \mathrm{CTT}_{2} \\
& \mathrm{CEX}_{\text {in }}=p_{1} \mathrm{CEX}_{1}+p_{2} \mathrm{CEX}_{2} .
\end{aligned}
$$


Clouds having CTHs of $11.25 \mathrm{~km}$ and $12.25 \mathrm{~km}$, and CEXs of -2.5 and -3 are examined, as shown in Fig. 6, taking various perturbations of the FOV. The full threeparameter retrieval algorithm is then applied to the inhomogeneous FOV radiances, the results of which are shown in Fig. 6.

In the first example, whereby the whole body of the cloud in the FOV has uniform extinction but varying CTHs, the CEX is very well retrieved. The effective CTH is retrieved to within $300 \mathrm{~m}$, although the CTT is less consistently retrieved. When there are near equal proportions of both clouds in the FOV, the retrieval struggles to converge, as noted in the open symbols in Fig. 6.

Next, taking a constant CTH across the FOV, but varying the CEX between -3 and -2.5 , generally the CEX is better retrieved for lower CEX than at the higher CEX end. The retrieved CEX is more-or-less proportional to the effective CEX in the FOV as the proportions of cloud shift - if not a little high in magnitude. Consequently, the CTH and CTT are retrieved representative of a lower cloud top than the effective values, although the agreement is better when more of the FOV is filled with optically thin cloud.

Finally, both the CTH and CEX are allowed to vary, and the CTT is varied in accordance with the simulated CTH for each cloud. Retrieved CTHs and CEXs are highly correlated with the effective CTHs and CEXs of the FOV, whilst the CTT is rather poorly retrieved, as it balances the two cloud types by consistently retrieving the unweighted average of the two CTTs of the two clouds.

\section{Case 2: vertical inhomogeneity}

In order to test how the retrieval responds to cloud which is not vertically homogeneous in the instrumental FOV, the specific case in which the vertical extent of the cloud is insufficient to fill the appropriate FOVs fully down to their bottommost extents are examined. This can be done by modifying the CFM for these purposes to simulate cloud having distinct cloud bottom heights $(\mathrm{CBH})$, by changing the bounds of the path or FOV convolution integrations to simulate these inhomogeneous cloudy states. To this end, cloud having CTH of $12.25 \mathrm{~km}$ and a CTT of $218.4 \mathrm{~K}$ are considered, having the $12 \mathrm{~km} \mathrm{FOV}$ as the FOV in which the cloud top occurs. CBHs of 6.5-11.5 km are imposed, for clouds having extinction coefficients between $10^{-3}-10^{-1} \mathrm{~km}^{-1}$. Thus, for the majority of CBHs, the lower FOV is not fully-filled vertically, and even the FOV in which the cloud-top is found is not filled for CBHs above $10 \mathrm{~km}$ (schematically shown in Fig. 7). The full three-parameter retrieval algorithm is then applied to CFM radiances, the results of which are shown in Fig. 7.

For clouds having extinction less than $10^{-2} \mathrm{~km}^{-1}$, CTHs are still retrieved to within about $70 \mathrm{~m}$, and the CTT is increased due partially to the anti-correlation between CTH and CTT, by less than a few degrees. Extinction values are decreased by roughly a half order of magnitude. Basically, the retrieval recognises that there is less radiance than there should be for a given set of macrophysical parameters, and attempts to fit less cloud, in both an optical and geometric sense, throughout the FOV.

For thicker clouds, the CTH is usually significantly decreased, and the retrieval attempts to balance CTT and CEX. Generally, CEX is overestimated - however there is little sensitivity to variations in CEX for such thick values of extinction, and hence the retrieved values are rather arbitrary.

Finally, the retrieval fails to converge for CFM-modelled clouds thinner than $1 \mathrm{~km}$, because this implies that the contribution to the bottom FOV is 0 by the cloud, and there is effectively one less measurement, since $\mathrm{CBH}$ is not parameterised. This may, in reality, be a critical point, as many high clouds (such as cirrus) can be vertically very thin.

\subsubsection{Using KOPRA simulations}

KOPRA simulations can be used to quantify, with some semblance to real scattering clouds, the effect of inhomogeneity on the retrieved parameters. Although it is impossible to quantify the errors coming from the infinite possible arrangements of inhomogeneous cloud in a MIPAS FOV, cases in which the CFM assumption of vertical homogeneity is violated are considered to exemplify the magnitude of errors introduced from the assumption of homogeneity. These errors can be extended to horizontal inhomogeneity, whereby the same proportion of the FOV is taken to be cloud-free over the path sampled by MIPAS - although it should be noted that there will exist differences, depending upon the nature of the horizontal inhomogeneity.

Only a small proportion of the KOPRA simulations prepared for the "MIPclouds" study actually satisfy the assumptions of homogeneity of the CFM. Cloud depths of $0.5 \mathrm{~km}$ through to $4 \mathrm{~km}$ are simulated - so there exist many cases in which the simulated cloud simply does not extend to the bottom of the considered $4 \mathrm{~km}$ MIPAS FOV. It is important to check how the retrieval model fares with respect to such cases, since it is likely that in real measurements there will frequently be cirrus which violate this assumption.

To this end, KOPRA simulations having a CTH of $12.5 \mathrm{~km}$ and cloud depths of $2.0 \mathrm{~km}, 3.0 \mathrm{~km}$ and $4.0 \mathrm{~km}$ are tested. These cloud depths represent fillings of the lowest FOV used in the measurement vector of between $6 \%$ and $65 \%$ of the FOV. Figure 8 shows the results of the retrieval algorithm applied to these simulations, where thin and thick clouds are said to be those having extinction coefficients along the limb path of less than and greater than $0.01 \mathrm{~km}^{-1}$, respectively.

The CTH continues to be well retrieved for thick clouds, as is the extinction for thin clouds. Again, it appears that the retrieval recognises thick cloud as such, but does not accurately reproduce the exact simulated value of extinction. The major discrepancy in the retrieved parameters is in the CTT, whereby it appears that the retrieval estimates the CTT about 

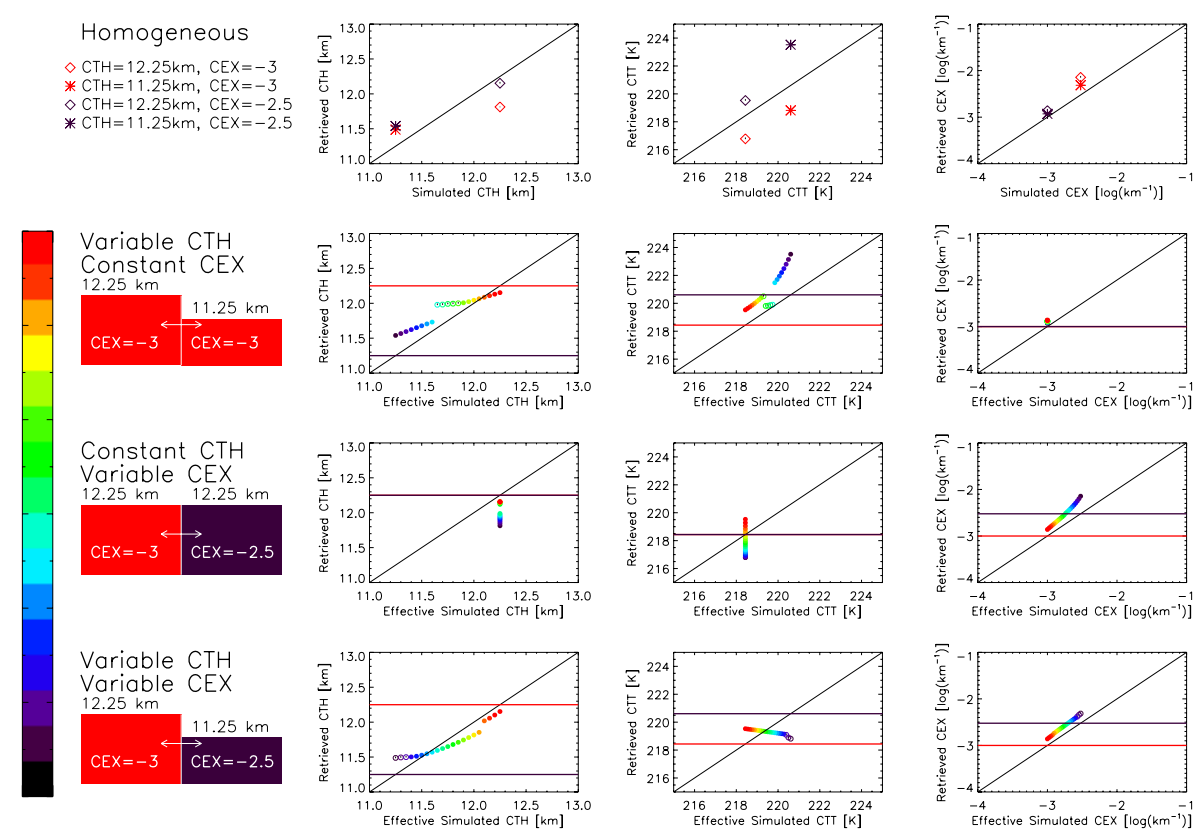

Fig. 6. For varying cases of FOV filling and inhomogeneity (first row = homogeneous; second row = constant $\mathrm{CEX}$, varying $\mathrm{CTH}$; third row = constant $\mathrm{CTH}$, varying CEX; final row = varying CEX and CTH), retrieved parameters of CTH (second column), CTT (third column) and CEX (last column) are plotted for varying proportions of filling by each cloud, from $100 \%$ the first cloud through to $100 \%$ the second cloud, with fraction varying linearly and indicated by colour. Filled circles mean retrieval has converged well - open circles mean it has been a retrieval with significant error.

$5 \mathrm{~K}$ too low: the retrieval acts to attribute the lower radiance coming from the simulations to a lowered CTT.

In the case that the cloud is only $2 \mathrm{~km}$ deep, the model underestimates the the extinction by half an order of magnitude, and increases the CTH. Cases having depths of 3-4 km have the parameters retrieved closer to those simulated, although the model sometimes compensates for the missing radiance by lowering the CTT by up to about $10 \mathrm{~K}$. The CFM/retrieval are probably "helped out" in a way because the effect of the extra radiation emitted by scattering clouds is partially cancelled out by the fact that there is simply not as much of the cloud as assumed. In any case, it appears that for inhomogeneous clouds having small cloud depths the retrieval somehow manages to still retrieve more-or-less fairly reasonable values of CTH, CTT and CEX, even though this may be a result of two CFM shortcomings partially cancelling each other out.

\subsection{Water vapour continuum}

At altitudes sampled by the lower tangent heights in the vertical MIPAS scan pattern (e.g. those less than about $6 \mathrm{~km}$ ), the water vapour continuum is difficult to distinguish from the continuum radiance introduced by emitting clouds. Thus, regions of large water vapour concentration could become a potential issue for reliable cloud detection, which could lead to statistical offsets in retrieved cloud products. Although this has not been studied in this work, in the current algorithm the absorption from the water vapour continuum is taken into account to some extent in the utilised molecular transmittance spectra, whereby the expected water vapour continuum is effectively "subtracted" from the measured continuum to establish the cloud contribution.

\subsection{Pointing error}

Pointing errors will make MIPAS tangent altitudes uncertain by several hundred metres - which is approximately the size of the largest uncertainties in the retrieved errors of CTH. The tangent altitudes are used only to help determine firstguesses for CTH and CTT, so as long as the a priori error ranges are set sufficiently large to encompass the range of pointing uncertainty (as they are in the current algorithm), errors in the pointing should not affect the final retrieved values of CTH and CTT.

\section{Application of algorithm}

This section shows the application of the described retrieval algorithm to a small set of MIPAS data (Sect. 4.1), in order to highlight the quantities and errors available from the retrieval process itself. It also qualitatively discusses the values retrieved by the algorithm applied to the MIPAS data to those reported by the Cloud-Aerosol Lidar and Infrared 

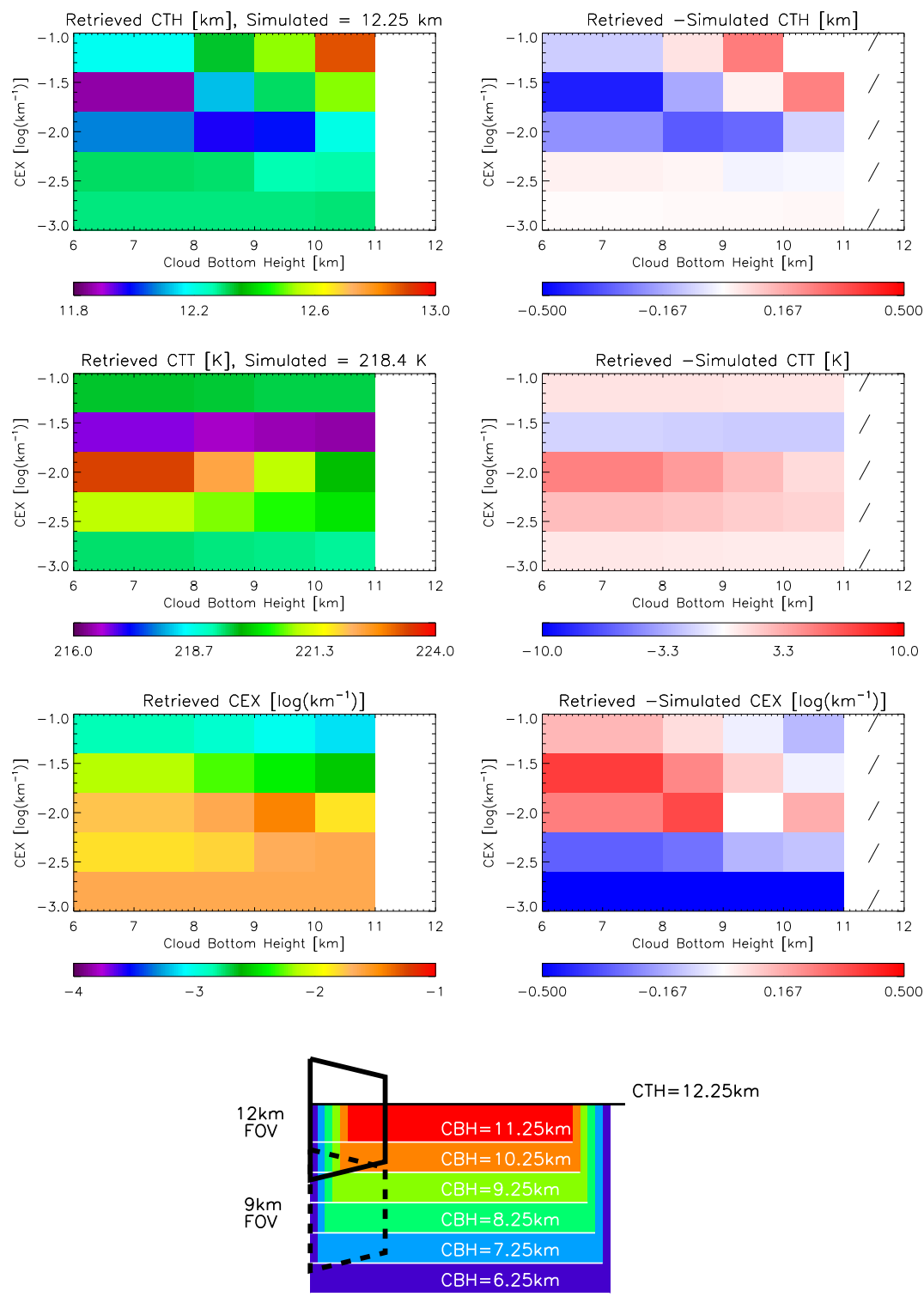

Fig. 7. Retrieved (first column) parameters of CTH (top row), CTT (second row) and CEX (third row), along with the difference between the retrieved and simulated parameters (second column) for CFM-simulated clouds which do not satisfy the CFM assumptions of vertical homogeneity, as varied by clouds of different CBHs and CEXs. Bottom plot shows schematic of the relative position of each sampled CBH, with respect to the $9 \mathrm{~km}$ and $12 \mathrm{~km}$ FOVs.

Pathfinder Satellite Observation (CALIPSO) cloud climatology for cloud top height (Winker et al., 2009), and the Polarization and Anisotropy of Reflectances for Atmospheric Sciences coupled with Observations from a Lidar (PARASOL) cloud opacity records (Leroy et al., 1997; Deschamps et al., 1994).

\subsection{Example results: 30 August 2009}

In this section, all measurements registered by MIPAS on 30 August 2009 have been processed using the described algorithm to highlight the products calculated and available for further analysis. Figure 9 shows the retrieved values of CTH, CTT and CEX, along with the errors stemming from the retrieval process itself (from the retrieval error covariance matrix). Furthermore, the types of retrieval, as discussed in Sect. 2.7, are identified by different symbols - and profiles in which there is deemed to be no cloud present are identified by a cross, giving an indication of the proportion of vertical scans taken through the atmosphere having cloud present somewhere in the scan.

The $\hat{\mathbf{S}}_{\mathbf{x}}^{\prime}$ errors are related to the type of retrieval carried out. For instance, for CTH and CTT, the lowest $\hat{\mathbf{S}}_{\mathbf{x}}^{\prime}$ error is 

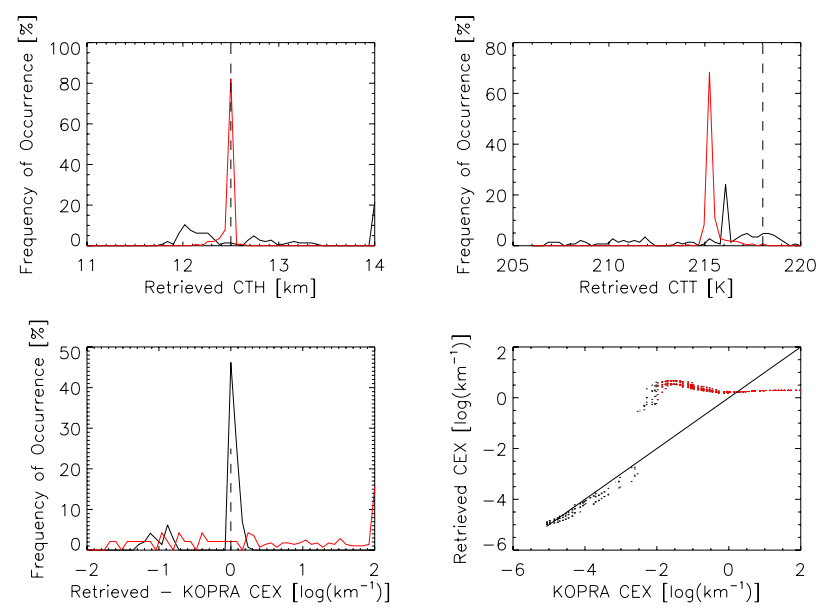

Fig. 8. In all plots, red is used to denote optically "thick" extinction simulations, and black for "thin" simulations. Top panels: PDFs of retrieved CTH (left) and CTT (right) for KOPRA-simulated clouds having $\mathrm{CTH}=12.5 \mathrm{~km}$. Dashed line indicates simulated "true" value. Lower left panel: PDF of the difference between KOPRAsimulated CEX and retrieved CEX. Lower right panel: scatterplot of retrieved CEX (right panel) for KOPRA-simulated clouds having $\mathrm{CTH}=12.5 \mathrm{~km}$. Black line shows one-to-one division.

obtained when retrieval Type 2 is used, which comes as no surprise since effectively the retrieval of CEX is removed, and hence two pieces of information are sought from two measurements. Along the same line of thought, retrieval Type 3 yields the worst errors for CTH and CTT, which is again expected, since there is effectively only one measurement from which to estimate the parameters. The Type 1 retrieval suffers from the same issue of under-determination as does the Type 3: although in this case, three parameters are sought from two radiance measurements and one pseudo-measurement of CEF. Figure 9 shows the distribution of these retrieval errors for the day's worth of MIPAS data. For the full three-parameter retrieval the errors due to the optimal estimations forward model/inversion are within $250 \mathrm{~m}$ for CTH, $3 \mathrm{~K}$ for CTT, and half an order of magnitude of the value of the extinction coefficient $k_{\mathrm{c}}$ along the limb path. Again, it must be emphasized that these errors in no way account for the real errors in the retrieved parameters, which are dominated by inadequacies in the forward model.

Typically less than $25 \%$ of sampled MIPAS scan profiles are cloud free throughout the atmosphere, about $40 \%$ of vertical scans are retrieved with the full Type 1 retrieval, whilst about $25 \%$ are retrieved with the Type 2 retrieval and about $10 \%$ with the Type 3 retrieval. The proportion of unsuccessful retrievals is less than $1 \%$.

Having introduced the products available from application of the retrieval algorithm to MIPAS data, a brief comparison to current CALIPSO and PARASOL/POLDER cloud products is presented, to preliminarily assess qualitatively whether it provides sensible estimates of cloud properties. This comparison is not meant as a rigorous algorithm/product validation.

It should be noted that CALIPSO infrared cloud products are determined from nadir-measurements, which introduces inherent geometrical differences between the two analyses. That said, since CALIPSO products are derived primarily using the lidar which has the same vertical sensitivity as MIPAS, the nadir/limb incompatibility should be negligible. This is confirmed in comparisons of HIRDLS and CALIPSO cloud products which suggest that CALIPSO is able to capture much of the same cloud as limb-viewing instruments (Massie, 2010) - and, at least on a statistical level, can be compared reasonably well with limb-viewing instruments. As well, CALIPSO has a much better horizontal resolution than with MIPAS so it is possible that CALIPSO may be able to detect low clouds near to high clouds which MIPAS would miss, thus potentially biassing the averaging statistics further.

CALIPSO's vertical feature mask differentiates between varying atmospheric states, including clear sky, cloud, aerosol, stratospheric layers, and regions whereby the lidar signal has totally attenuated (Winker et al., 2009). For the purposes of this preliminary comparison, each lidar crosssection of the atmosphere is assessed to find the highest occurring cloud top, which is then reported as the cloud top height at that geographical location. This should mimic what MIPAS would measure, as infrared limb-viewers cannot see below the highest cloud layer of non-negligible opacity. Furthermore, flags for stratospheric layers as well as aerosols are considered, as it is possible that these may be selected as thin clouds by the presented algorithm (Fig. 10).

It should be noted that CALIPSO and MIPAS do not make coincident measurements, as demonstrated by the more than three-hour difference in equator-crossing time between the two - so for a rigorous validation, a full statistical treatment of several months of data should be analysed. For the purposes of this comparison, however, only the general large-scale features and trends are sought. For this very limited selection of data, generally CALIPSO and MIPAS cloud top heights are similar, with both highlighting expected behaviour: increased cloud top heights approaching the equator and tropics, mirroring the heightened tropopause; heightened cloud top heights over the Indonesian Toga Core; obvious north/south asymmetry, with lower cloud top heights in the Arctic than those over the Antarctic; and clear polarstratospheric cloud activity over the Antarctic, which is ubiquitous at that time of year. For cloud having cloud top heights greater than $10 \mathrm{~km}$, generally both CALIPSO and MIPAS show cloud top heights agreeing within $1 \mathrm{~km}$ for nearby geographical regions, although it must be noted that these cannot be asserted to actually be coincident measurements due to the differences in measurement times. For lower clouds, there is more variation, which is at least partially attributable to the fact that CALIPSO measures right down to the ground, whereas MIPAS can measure cloud top heights no lower than 

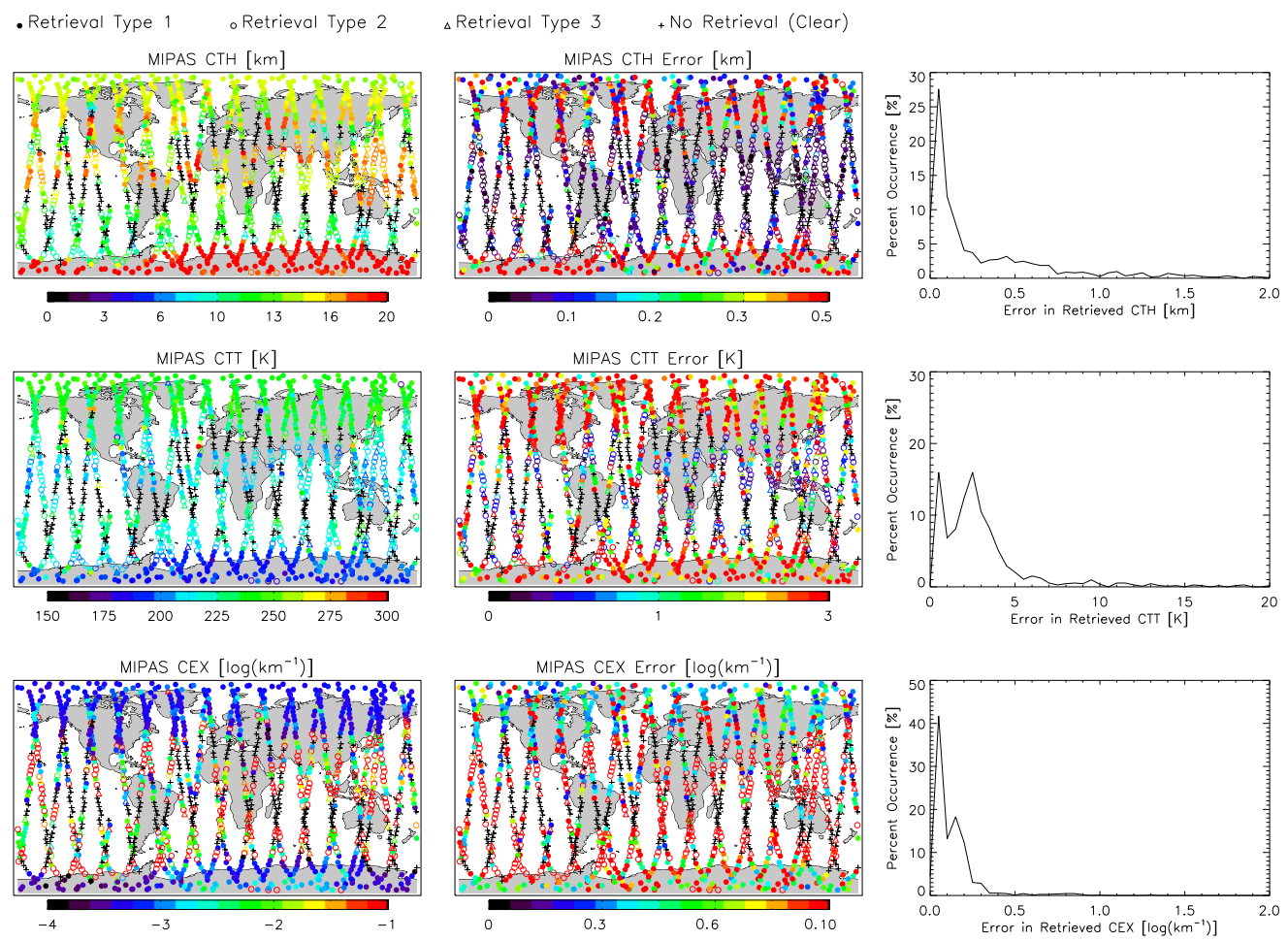

Fig. 9. Application of algorithm to all MIPAS measurements taken on 30 August 2009. Retrieved parameters (left column) of CTH (top panels), CTT (middle panels) and CEX (bottom panels) and errors thereof (middle panels) are given, noting the type of retrieval, corresponding to the available measurement FOVs. Right panels show probability distribution functions of the retrieval errors from $\hat{\mathbf{S}}_{\mathbf{x}}^{\prime}$ corresponding to CTH, CTT and CEX.

its lowest FOV, which itself is latitude-dependent. The MIPAS points in Fig. 10 having $\mathrm{CTH}=0 \mathrm{~km}$ are therefore profiles of atmosphere having no cloud detected. A full comparison of polar stratospheric clouds as measured by MIPAS and CALIPSO has been carried out by Höpfner et al. (2009).

It appears that the CEF detection method identifies what CALIPSO masks as stratospheric layers as cloud, given the reported heights of CALIPSO's clouds and stratospheric layers. It is likely that the stratospheric hazes over the South Pole are indeed polar stratospheric clouds, so this is an acceptable function of the used CEF method. The stratospheric layers identified by CALIPSO over northern Europe, northern Asia and northern North America are also most likely identified as cloud by the CEF method, given the similar altitudes of the features. Arguably, aerosol layers are a limit to very thin, diffuse clouds, although formed of different particles. So whilst it is concerning that aerosol layers can be identified as cloud, it is unsurprising as both have similar effects on spectra. Both aerosols and clouds will be inherently problematic for spectrally-based detection methods, such as CEF and CI. However, except for cases of stratospheric aerosols or hazes, most layers are expected to occur below the lowest tangent height measured by MIPAS, and will not be accounted for in cloud climatologies thus com- piled. Beyond this, no conclusion can be reached concerning misidentification of aerosol (as classified by CALIPSO) as cloud by MIPAS, as in most cases, the top of the aerosol layer is below the lowest altitude range sampled by MIPAS.

PARASOL/POLDER is used to qualitatively assess the reported values of cloud extinction, although for the sake of brevity these are not shown here. Again, it must be noted that the measurements are not coincident, and in order to make any real comparison, a rigorous statistical treatment must be taken. Nevertheless, again general trends and behaviour can be assessed, by comparing where each instrument sees optically "thick" and "thin" cloud, especially since the geometry of the PARASOL/POLDER optical depth is not the same as that of the MIPAS-derived extinction coefficient. Comparison of cloud opacities is only possible for those MIPAS retrievals for which a full three-parameter retrieval (Type 1) is possible. Given these caveats, for the limited dataset analysed generally close geographical regions tend to have similar relative opacities measured by both PARASOL and MIPAS, in the sense of "thick" versus "thin" cloud - however, the opacity field is quite localised and variable, so general conclusions are difficult to make from a geographical standpoint. 

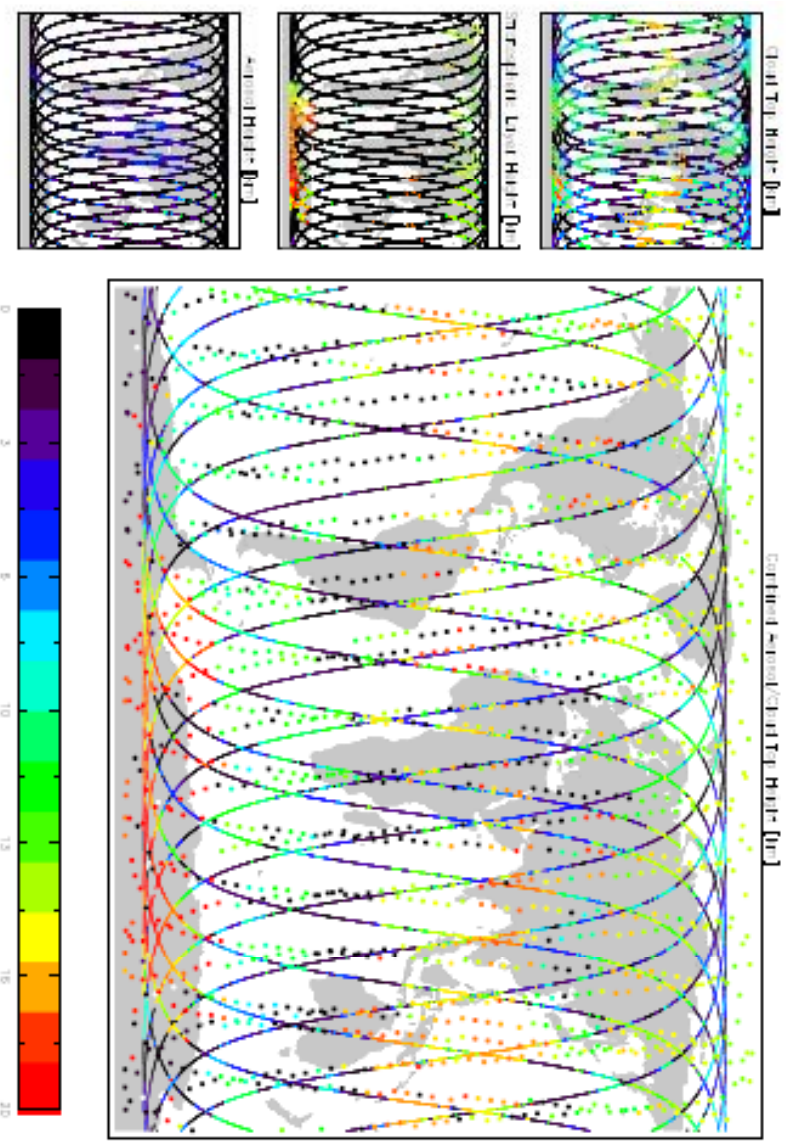

Fig. 10. Comparison of CALIPSO vertical feature mask (solid lines) and MIPAS cloud top heights (dots) on 30 August 2009. In left column of panels, the heights of the highest occurring cloud, stratospheric layer, or aerosol at each geographical location measured by CALIPSO are given. In the big panel, the highest occurring altitude of the three feature masks is given as the combined "cloud" top height for CALIPSO, and the MIPAS cloud top heights are overlaid as dots.

\subsection{Comparison of CEF and CI detection mechanisms}

Section 2.3 describes the method used to select measurements as containing cloud and as the CI Method is the traditionally used method, this section seeks to assert that the CEF is reasonable as a cloud detection method, and in fact, may capture more optically-or-geometrically thin cloud. In this section, both CEF and CI cloud detection methods are applied to the same set of spectra. This set of spectra is selected as all those spectra measured below $30 \mathrm{~km}$ and above the tangent height which the CEF method first detects a cloud top, which will give a realistic selection of clear and cloudy examples. Comparison between the two detection mechanisms is made using real MIPAS data for all measurements registered on 30 August 2009.

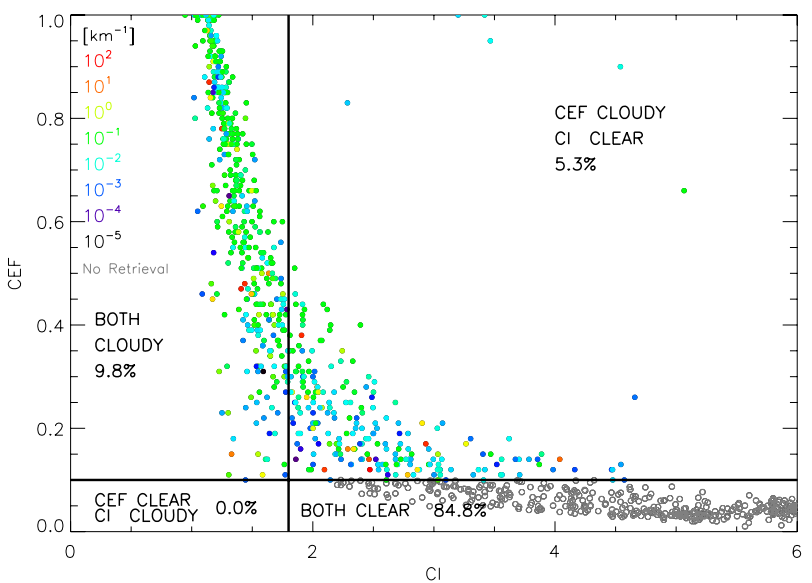

Fig. 11. Correlation between $\mathrm{CI}$ and CEF (evaluated in MW1) colour-coded by extinction coefficient (for those cases for which the retrieval has been evaluated) and by open circles for clear scans, for all spectra above the cloud top (if any) measured MIPAS on 30 August 2009. CI is anti-correlated and CEF is correlated with cloud amount. Horizontal line shows the CEF threshold (above which cloud occurs) and vertical line shows the CI threshold (to the left of which cloud occurs).

Figure 11 shows the results of this comparison, highlighting that the CEF scheme detects more cloud than does the CI method with the application of the operational CI threshold of 1.8 (e.g. Spang, 2004) and CEF threshold of 0.1. It should be noted that the CI threshold of 1.8 is operationally set to detect clouds sufficiently opaque to cause problems for trace gas retrievals. It is plausible that the scatter of points at higher CI are indeed cloudy cases, as there appears to be larger scatter than attributable to normal variations of temperature and trace-species concentrations. Furthermore, if the thresholds are applied and cloud detection is carried out, the CEF scheme detects more cloud particularly in regions where thin cloud such as polar stratospheric clouds or cirrus are expected. In general, the CEF method selects far more measurements as cloud-contaminated - which should yield a more complete selection of cloud data upon which to create climatological analysis.

It is worth noting that the percentage of spectra identified as containing cloud is dependent upon the choice of threshold applied to each detection method. For instance, at the operation threshold of 1.8 , the CI method detects cloud in $9.8 \%$ of the studied set of spectra. The CEF method will select $9.8 \%$ of the spectra as containing cloud if its threshold is modified to 0.32 (instead of the suggested 0.1), although it is worth noting that both methods do not choose all the same individual cases as cloud-contaminated. If the CI threshold for cloud is loosened to 4.0 , it selects $17.6 \%$ of the spectra in the set as cloudy - a percentage which can be matched by setting the CEF threshold to 0.08 . 


\section{Conclusions}

This study details an algorithm for modelling cloud top height, cloud top temperature and extinction coefficient using a simple non-scattering model in a manner suitable for an operational processor. As such, a couple of major assumptions, namely homogeneity and a non-scattering atmosphere, have been taken, which have implications on the accuracy of the final retrieved parameters, and which are the dominant sources of error in the retrieved parameters.

The retrieval algorithm has been applied to singlescattering simulations for which the assumption of homogeneity is satisfied - and in this case, it appears that cloud top height and cloud top temperature can generally be successfully retrieved to within $250 \mathrm{~m}$ and $3 \mathrm{~K}$, and half an order of magnitude of the extinction coefficient, although it is important to bear in mind that these values do not represent anything near the real error range when the algorithm is applied to real measurements for which the assumptions of homogeneity almost certainly will not be met. This can be used to assess how well the retrieval model can fit realistic clouds which satisfy the homogeneity assumption, within the extinction range of $10^{-4} \mathrm{~km}^{-1}$ and $10^{-2} \mathrm{~km}^{-1}$, for which there is sensitivity to extinction and the process of scattering is not the dominant radiative process. It should be noted, though, at this point that an operational processor cannot really carry out scattering calculations due to the computational expense in analysis of large datasets.

The greatest error is expected to result from the error in the initial forward model assumption of homogeneity - that is, that a cloud can be represented by a single flat cloud top height, a single extinction coefficient and a consistent temperature structure throughout the body of the cloud. Homogeneity is a simplification of the geometry and optics of real clouds - but there are infinite possible cloud fields and it is impossible to retrieve inhomogeneous fillings of the MIPAS FOV without prior knowledge of the geometry of the inhomogeneity, such as was done in Rydberg et al. (2009) for Odin/SMR cloud retrievals which used extra information from CloudSat imagery. It could be argued that a statistically-obtained "average inhomogeneous" filling of the FOV would be a better approximation, as the assumption of homogeneity is insufficient to fully represent reality, and really represents an extreme case in the range of possible cloud fields. Homogeneity is used in this work for simplicity's sake alone, and the nature of the effect this assumption has on the retrieved parameters has been preliminarily discussed in Sect. 3.4. A natural development of the current model would be to extend it to better describe inhomogeneous fillings of the FOV, without need for added a priori knowledge garnered from another instrument (such as a limb imager coinciding with the fourier transform spectrometer view).

The CFM in conjunction with the single-scattering simulations have been used to preliminarily study the effect of inhomogeneity on the current retrieved products - although this is difficult to quantify, given the infinite possible combinations of inhomogeneities, and the added complications arising from scattering, due to the large range of microphysical parameters which contribute. Generally, the retrievals are quite robust, however the retrieved parameters are especially sensitive to the assumptions of homogeneity within the FOV for thick cloud for which there are significant radiance discrepancies between measurement and forward model. It is possible, as well, for optically or geometrically thin $(<1 \mathrm{~km})$ cloud, that the CEX retrieval can be affected by atmospheric contributions along the limb path over which the extinction is calculated, although this effect is hopefully minimised inasmuch as possible by using atmospheric windows of negligible gaseous absorption. This, may in fact, be an important point, as cirrus fields can frequently be vertically thin.

Furthermore, since the CEF detection method has apparent problems differentiating between high cloud and stratospheric aerosol layers some care must be thus taken when the algorithm is applied to real data so that such layers are properly identified and not averaged into cloud products and statistics.

Taking all these difficulties and model insufficiencies into account, the algorithm seems able to estimate relatively accurate CTHs and CTTs - although it is impossible to give a quantitative measure of the accuracy due to the assumptions of homogeneity and non-scattering being insufficient to represent real clouds. Conservatively, the algorithm is able to retrieve CTH and CTT to sub-tangent-altitude spacing - which represents an improvement on previous limb-sounder cloud top retrievals, which are predominately based on detection of cloud. The values of extinction must be used qualitatively to assess the overall cloud optical thickness - be it thick or thin - rather than as a quantitative measure of extinction coefficient, since scattering is not included.

Acknowledgements. Part of this work was done as part of a DPhil undertaken at the University of Oxford under the funding of the UK Commonwealth Scholarship Committee. Part of this work was supported by ESA through the MIPClouds project: "Cloud Information Retrieval from MIPAS Measurements", $\mathrm{AO} / 1-5255 / 06 / \mathrm{I}-\mathrm{OL}$.

Edited by: P. Eriksson

\section{References}

Barton, I.: Upper-level cloud climatology from an orbiting satellite, J. Atmos. Sci., 40, 435-447, 1983.

Bernath, P.: The Atmospheric Chemistry Experiment (ACE): An Overview, Geoscience and Remote Sensing Symposium, IGARSS '02, 2002.

CLAES: website on SPASCI CLAES Mission, www.spasci.com/ CLAES/mission.html, 2007.

Deschamps, P. Y., Breon, F. M., Leroy, M., Podaire, A., Bricaud, A., Buriez, J. C. and Seze, G.: The POLDER mission: Instrument 
characteristics and scientific objectives, IEEE T. Geosci. Remote, 32, 598-615, 1994.

Dudhia, A.: website on the Reference Forward Model (RFM): Software User's Manual (SUM), http://www.atm.ox.ac.uk/RFM/ sum/, 2005.

Dudhia, A., Jay, V. L., and Rodgers, C. D.: Microwindow selection for high-spectral-resolution sounders, Appl. Optics, 41, 36653673, 2002.

European Space Agency: website on ESA ENVISAT, http://envisat. esa.int/instruments/images/MIPAS_Interferometer.gif, 2005.

ESA Living Planet: website, http://www.esa.int/esaLP/ SEMNAT8I77G_LPcampaigns_0.html, 2010.

Ewen, G.: Infrared Limb Observations of Cloud, DPhil thesis in Atmospheric, Oceanic and Planetary Physics, University of Oxford, Oxford, UK, 2005.

Fougnie, B., Bracco, G., Lafrance, B., Ruffel, C., Hagolle, O. and Tinel, C.: PARASOL in-flight calibration and performance, Appl. Optics, 46(22), 5435-5451, 2007.

Gilbert, K., Turnbull, D., Walker, K., Boone, C., McLeod, S., Butler, M., Skelton, R., Bernath, P., Chateauneuf, F., and Soucy, M.-A.: The onboard imagers for the Canadian ACE SCISAT-1 mission, J. Geophys. Res., 112, D12207, doi:10.1029/2006JD007714, 2007.

Global Change Master Directory: website on ISAMS, http://geodiscover.cgdi.ca/gdp/search?action= fullMetadata $\backslash$ \&entryLang=fr $\backslash$ \&entryId=5640 $\backslash$ \&entryType $=$ productCollection, 2007.

Hervig, M. and Deshler, T.: Evaluation of aerosol measurements from SAGE II, HALOE, and balloonborne optical particle counters, J. Geophys. Res., 107, AAC3.1-AAC3.12, 2002.

Hervig, M., Deshler, T., and Russell, J.: Aerosol size distributions obtained from HALOE spectral extinction measurements, J. Geophys. Res., 103, 1573-1583, 1998.

Höpfner, M. and Emde, C.: Comparison of single and multiple scattering approaches for the simulation of limb-emission observations in the mid-IR, J. Quant. Spectrosc. Ra., 91, 275-285, 2005.

Höpfner, M., Pitts, L., and Poole, J.: Comparison between CALIPSO and MIPAS observations of polar stratospheric clouds, J. Geophys. Res., 114, D00H05, doi:10.1029/2009JD012114, 2009.

Hurley, J., Dudhia, A., and Grainger, R. G.: Cloud detection for MIPAS using singular vector decomposition, Atmos. Meas. Tech., 2, 533-547, doi:10.5194/amt-2-533-2009, 2009.

IMK: website on IMK Karlsruhe Optimized and Precise Radiative transfer Algorithm, http://www-imk.fzk.de/asf/ame/ publications/kopra_docu/, 2008.

Intergovernmental Panel on Climate Change: Fourth Assessment Report Climate Change 2007 - The Physical Science Basis, Cambridge University Press, Cambridge, 2008.

ISCCP: website on ISCCP, http://isccp.giss.nasa.gov/index.html, 2008.

ISCCP: website on ISCCP algorithm description, http://isccp.giss. nasa.gov/newalg.html, 2006.

Kahn, B., Eldering, A., Irion, F., Mills, F., Sen, B., and Gunson, M.: Cloud identification in Atmospheric Trace Molecule Spectroscopy infrared occultation measurement, Appl. Optics, 41, 2768-2780, 2002.

Khosravi,R., Lambert, A., Lee, H., Gille, J., Barnett, J., Francis, G., Edwards, D., Halvorson, C., Massie, S., Craig, C.,
Krinsky, C., McInerney, J., Stone, K., Eden, T., Nardi, B., Hepplewhite, C., Mankin, W., and Coffey, M.: Overview and characterization of retrievals of temperature, pressure, and atmospheric constituents from the High Resolution Dynamics Limb Sounder (HIRDLS) measurements, J. Geophys. Res., 114, D20304, doi:10.1029/2009JD011937, 2009.

King, M.D., Platnick, S., Wind, G., Arnold, G.T., Dominguez, R.T.: Remote sensing of radiative and microphysical properties of clouds during TC4: Results from MAS, MASTER, MODIS, and MISR, J. Geophys. Res., 115, D00J07, doi:10.1029/2009JD013277, 2010.

Lambert, A., Bailey, B., Edwards, D., Gille, J., and Johnson, B.: High Resolution Dynamics Limb Sounder Level-2 Algorithm Theoretical Basis Document 2, Tech. rep., 1999.

Leroy, M., Deuze, J. L., Bréon, F. M., Hautecoeur, O., Herman, M., Buriez, J. C., Tanre, D., Bouffies, S., Chazette, P., and Roujean, J. L.: Retrieval of atmospheric properties and surface bidirectional reflectances over the land from POLDER, J. Geophys. Res., 102, 17023-17037, 1997.

Mantovani, R.: ENVISAT MIPAS Report: March 2004-February 2005, ENVI-SPPA-EOPG-TN-05-0006, Tech. rep., European Space Agency, 2005.

Massie, S. T., Gille, J., Khosravi, R., Lee, H., Kinnison, D., Francis, D., Nardi, B., Eden, T., Craig, C., Halvorson, C., Coffey, M., Packman, D., Cavanaugh, C., Craft, J., Dean, V., Ellis, D., Barnett, J., Hepplewhite, C., Lambert, A., Manney, G., Strawa, A., and Legg, M.: High Resolution Dynamics Limb Sounder observations of polar stratospheric clouds and subvisible cirrus, J. Geophys. Res., 112, D24S31, doi:10.1029/2007JD008788, 2007.

Massie, S. T., Gille, J., Craig, C., Khosravi, R., Barnett, J., Read, W., and Winker, D.: HIRDLS and CALIPSO observations of tropical cirrus, J. Geophys. Res., 115, D00H11, doi:10.1029/2009JD012100, 2010.

Mendrok, J., Schreier, F., and Höpfner, M.: Estimating cirrus cloud properties from MIPAS data, Geophys. Res. Lett., 34(8), L08807, doi:10.1029/2006GL028246, 2007.

Mergenthaler, J., Kumer, J., and Roche, A.: CLAES southlooking aerosol observations for 1992, Geophys. Res. Lett., 20(12), 1295-1298, 1993.

Moore, D.: website on University of Leicester Cloud Top Height product, http://www.leos.le.ac.uk/mipas/data/nrt_ci.html, 2008.

Murtagh, D., Frisk, U., Merino, F., Ridal, M., Jonsson, A., Stegman, J., Witt, G., Eriksson, P., Jimenez, C., Megie, G., de La Noe, J., Ricaud, P., Baron, P., Pardo, J. R., Hauchcorne, A., Llewellyn, E. J., Degenstein, D. A., Gattinger, R. L., Lloyd, N. D., Evans, W. F. J., McDade, I. C., Haley, C., Sioris, C., von Savigny, C., Solheim, B. H., McConnell, J. C., Strong, K., Richardson, E. H., Leppelmeier, G. W., Kyro a, E., Auvinen, H., and Oikarinen, L.: An overview of the Odin atmospheric mission, Can. J. Phys., 80, 309-319, 2002.

NASA: website on GES Distributed Active Archive Center LIMS User's Guide, ftp://disc1.sci.gsfc.nasa.gov/data/lims/ Documentation/, 2007.

Prabhakara, C., Fraser, R., Dalu, G., Wu, M. abd Curran, R., and Styles, T.: Thin cirrus clouds: Seasonal distribution over oceans deduced from NIMBUS-4 IRIS, J. Appl. Meteor., 27, 379-399, 1988.

Remedios, J.: Profiles for MIPAS, EOS, Space Research Centre, 
Leicester, UK, January 2001.

Rodgers, C.: Inverse Methods for Atmospheric Sounding: Theory and Practice, World Scientific Publishing Co Pte Ltd., 2000.

Rydberg, B., Eriksson, P., Buehler, S. A., and Murtagh, D. P.: NonGaussian Bayesian retrieval of tropical upper tropospheric cloud ice and water vapour from Odin-SMR measurements, Atmos. Meas. Tech., 2, 621-637, doi:10.5194/amt-2-621-2009, 2009.

SAGE-III-ATBD-Team: SAGE III Algorithm Theoretical Basis Document (ATBD) Cloud Data Products, LaRC 475-00-106, 1.2, Tech. rep., 2002.

Spang, R., Remedios, J., and Barkley, M.: Colour Indices for the Detection and Differentiation of Cloud Types in Infra-red Limb Emission Spectra, Adv. Space Res., 33, 1041-1047, 2004.

Spang, R., Griessbach, S., Hopfner, M., Dudhia, A., Hurley, J., Siddans, R., Waterfall, A., Remedios, J., and Sembhi, H.: Technical Note: Retrievability of MIPAS cloud parameter, ESA-ITT AO/15255/06/I-OL, Tech. rep., European Space Agency, 2008.

Stephens, G. L., Vane, D. G., Boain, R. J., Mace, G. G., Sassen, K., Wang, Z., Illingworth, A. J., O'Connor, E. J., Rossow, W. B., Durden, S. L., Miller, S. D., Austin, R. T., Benedetti, A., Mitrescu, C., and the CloudSat Science Team: The CloudSat mission and the A-TRAIN: A new dimension to space-based observations of clouds and precipitation, B. Am. Meteorol. Soc., 83, 1771-1790, 2002.

Stubenrauch, B., Kinne, R., and the GEWEX Cloud Assessment Team: Assessment of global cloud climatologies, GEWEX News, 19, http://www.gewex.org/images/Feb2009.pdf, 2009.

Taylor, F., Rodgers, C., Whitney, J., Werrett, S., Barnett, J., Peskett, G., Venters, P., Ballard, J., Palmer, C., Knight, R., Morris, P., Nightinggale, T., and Dudhia, A.: Remote Sensing of Atmospheric Structure and Composition by Pressure Modulator Radiometry From Space: The ISAMS Experiment on UARS, J. Geophys. Res., 98(6), 10799-10814, 1993.
Thomas, G. E., Poulsen, C. A., Siddans, R., Sayer, A. M., Carboni, E., Marsh, S. H., Dean, S. M., Grainger, R. G., and Lawrence, B. N.: Validation of the GRAPE single view aerosol retrieval for ATSR-2 and insights into the long term global AOD trend over the ocean, Atmos. Chem. Phys., 10, 4849-4866, doi:10.5194/acp-10-4849-2010, 2010.

Warren, S., Hahn, C., and London, J.: Simultaneous occurrence of different cloud types, J. Clim. Appl. Meteot., 24, 658-667, 1985.

Winker, D. M, Vaughan, M. A., Omar, A., Hu, Y., Powell, K. A., Liu, Z., Hunt, W. H., and Young, S. A.: Overview of the CALIPSO mission and the CALIOP data processing algorithms, J. Atmos. Ocean. Tech., 26, 2310-2322, 2009.

Woodbury, G. and McCormick, M.: Global Distributions of Cirrus Clouds Determined from SAGE Data, Geophys. Res. Lett., 10, 1180-1183, 1983.

Wu, D. L., Pickett, H. M. and Livesey, N. J.: Aura MLS THz observations of global cirrus near the tropopause, Geophys. Res. Lett., 35, L15803, doi:10.1029/2008GL034233, 2008.

Wylie, D. and Menzel, W.: Two years of cloud cover statistics using VAS, J. Clim. Appl. Meteor., 2, 380-392, 1989.

Wylie, D., Menzel, W., Woolf, H., and Strabala, K.: Four Years of Global Cirrus Cloud Statistics Using HIRS, J. Climate, 7(12), 1972-1986, 1994.

Wylie, D., Jackson, D., Menzel, W., and Bates, J.: Trends in Global Cloud Cover in Two Decades of HIRS Observations, J. Climate, 130, 3021-3033, 2005. 